\title{
Tests of General Relativity with GW170817
}

\author{
B. P. Abbott et al. \\ (LIGO Scientific Collaboration and Virgo Collaboration)
}

(Received 20 November 2018; revised manuscript received 21 March 2019; published 1 July 2019; corrected 20 August 2019)

\begin{abstract}
The recent discovery by Advanced LIGO and Advanced Virgo of a gravitational wave signal from a binary neutron star inspiral has enabled tests of general relativity (GR) with this new type of source. This source, for the first time, permits tests of strong-field dynamics of compact binaries in the presence of matter. In this Letter, we place constraints on the dipole radiation and possible deviations from GR in the post-Newtonian coefficients that govern the inspiral regime. Bounds on modified dispersion of gravitational waves are obtained; in combination with information from the observed electromagnetic counterpart we can also constrain effects due to large extra dimensions. Finally, the polarization content of the gravitational wave signal is studied. The results of all tests performed here show good agreement with GR.
\end{abstract}

DOI: 10.1103/PhysRevLett.123.011102

Introduction.-On August 17, 2017 at 12:41:04 UTC, the Advanced LIGO and Advanced Virgo gravitationalwave $(\mathrm{GW})$ detectors made their first observation of a binary neutron star inspiral signal, called GW170817 [1]. Associated with this event, a gamma ray burst [2] was independently observed, and an optical counterpart was later discovered [3]. In terms of fundamental physics, these coincident observations led to a stringent constraint on the difference between the speed of gravity and the speed of light, allowed new bounds to be placed on local Lorentz invariance violations, and enabled a new test of the equivalence principle by constraining the Shapiro delay between gravitational and electromagnetic radiation [2]. These bounds, in turn, helped to strongly constrain the allowed parameter space of alternative theories of gravity that offered gravitational explanations for the origin of dark energy [4-10] or dark matter [11].

In this paper we present a range of tests of general relativity (GR) that have not yet been done with GW170817. Some of these are extensions of tests performed with previously discovered binary black hole coalescences [12-18], an important difference being that the neutron stars' tidal deformabilities need to be taken into account in the waveform models. The parameter estimation settings for this analysis broadly match with those of Refs. [19,20], which reported the properties of the source GW170817. Our approach here is theory-agnostic where, using GW170817, we constrain generic features in the gravitational waveform that may arise from a breakdown of GR. For a detailed discussion about specific alternative theories that predict one or more of the physical effects discussed here see, for instance, Sec. 5 of Ref. [21] and Sec. 2 of Ref. [22].

*Full author list given at the end of the article.
Three types of tests are presented. First, we study the general-relativistic dynamics of the source, in particular constraining dipole radiation in the strong-field and radiative regime and checking for possible deviations in the post-Newtonian (PN) description of binary inspiral by studying the phase evolution of the signal. Next, we focus on the way gravitational waves propagate over large distances. Here we look for anomalous dispersion, which enables complementary bounds on violations of local Lorentz invariance to those of [2]; constraints on large extra spatial dimensions are obtained by comparing the distance inferred from the GW signal with the one inferred from the electromagnetic counterpart. Finally, constraints are placed on alternative polarization states, where this time the position of the source on the sky can be used, again because of the availability of an electromagnetic counterpart. We end with a summary and conclusions.

Constraints on deviations from the general-relativistic dynamics of the source.-Testing GR via the dynamics of a binary system involves constructing a waveform model that allows for parametrized deformations away from the predictions of GR and then constraining the associated parameters that govern those deviations [13,15,16,23-28]. For previous observations of coalescing binary black holes $[13,15]$, these tests relied on the frequency domain IMRPhenomPv2 waveform model of Refs. [29-31], which describes the inspiral, merger, and ringdown of vacuum black holes, and provides an effective description of spin precession, making the best use of the results from analytical and numerical relativity [32-39]. The phase evolution of this waveform is governed by a set of coefficients $p_{n}$ that depend on the component masses and spins. These coefficients include post-Newtonian (PN) parameters and phenomenological constants that are calibrated against numerical relativity waveforms to describe the intermediate regime between inspiral and 
merger, as well as the merger and ringdown. To test GR, the waveform model is generalized to allow for relative deviations in each of the coefficients in turn, i.e., by replacing $p_{n} \rightarrow\left(1+\delta \hat{p}_{n}\right) p_{n}$, where the $\delta \hat{p}_{n}$ are zero in GR. The $\delta \hat{p}_{n}$ are then varied along with all the parameters that are also present in the case of GR (masses, spins, and extrinsic parameters), and posterior density functions (PDFs) are obtained using LALInference [40]. For GR to be correct, the value $\delta \hat{p}_{n}=0$ should fall within the support of each of the PDFs. Note that although one could also let all of the testing parameters vary at the same time, this will tend to lead to uninformative posteriors (see, e.g., Ref. [13]). Fortunately, as demonstrated in Refs. [27,28,41,42], checking for a deviation from zero in a single testing parameter is an efficient way to uncover GR violations that occur at multiple PN orders, and one can even find violations at powers of frequency that are distinct from the one that the testing parameter is associated with $[27,28,42]$. Thus, such analyses are well suited to search for generic departures from GR. There is a limitation though: Should a GR violation be present, then the measured values of the $\delta \hat{p}_{i}$ will not necessarily reflect the predictions of whichever alternative theory happens to be the correct one. To reliably constrain theory-specific quantities such as coupling constants or extra charges, one should utilize full inspiral waveform models from specific modified gravity theories, including modifications to the phase at all the orders where they appear. Unfortunately, in most cases only leading-order modifications are available at the present time [43]. However, in this section the focus is mostly on model-independent tests of general relativity itself.

In this work, we modify this approach in two ways. First, we use waveform models more suitable for binary neutron stars. Second, whereas the infrastructure [27] used to test GR with binary black holes observations [13,15] was restricted to waveform models that depend directly on the coefficients $p_{n}$, we also introduce a new procedure that can include deviations to the phase evolution parametrized by $\delta \hat{p}_{n}$ to any frequency domain waveform model. We conduct independent tests of GR using inspiral-mergerringdown models that incorporate deviations from GR using each of these two prescriptions; by comparing these analyses, we are able to estimate the magnitude of systematic modeling uncertainty in our results.

The merger and ringdown regimes of binary neutron stars differ from those of binary black holes, and tidal effects not present in binary black holes need to be included in the description of the inspiral. Significant work has been done to understand and model the dynamics of binary neutron stars analytically using the PN approximation to general relativity [44]. This includes modeling the nonspinning [32,33] and spinning radiative (or inspiral) dynamics [34-39] as well as finite size effects [45-47] for binary neutron star systems. Frequency domain waveforms based on the stationary phase approximation [48] have been developed incorporating the above-mentioned effects [49-51] and have been successfully employed for the data analysis of compact binaries. A combination of these analytical results with the results from numerical relativity simulations of binary neutron star mergers (see Ref. [52] for a review) have led to the development of efficient waveform models which account for tidal effects [53-55].

We employ the NRTidal models introduced in Refs. [55,56] as the basis of our binary neutron star waveforms: frequency domain waveform models for binary black holes are converted into waveforms for inspiraling neutron stars that undergo tidal deformations by adding to the phase an appropriate expression $\phi_{T}(f)$ and windowing the amplitude such that the merger and ringdown are smoothly removed from the model; see Ref. [56] for details. The closed-form expression for $\phi_{T}(f)$ is built by combining PN information, the tidal effective-one-body (EOB) model of Ref. [53], and input from numerical relativity (NR). We consider two waveform models that use this description of tidal effects. One of these modelsIMRPhenOmPNRT, detailed below-describes a binary neutron star with precessing spins. Though the form of $\phi_{T}(f)$ was originally obtained in a setting where the neutron stars were irrotational or had their spins aligned to the angular momentum, tides can be included in this waveform model by first applying $\phi_{T}(f)$ to an aligned-spin waveform, and then performing the twisting-up procedure that introduces spin precession [57].

The first binary neutron star model we consider is constructed by applying this procedure to IMRPhenomPv2 waveforms. Following the nomenclature of Ref. [19], we refer to the resulting waveform model as PhenomPNRT. Parametrized deformations $\delta \hat{p}_{n}$ are then introduced as shifts in parameters describing the phase in precisely the same way as was done for binary black holes. This will allow us to naturally combine PDFs for the $\delta \hat{p}_{n}$ from measurements on binary black holes and binary neutron stars, arriving at increasingly sharper results in the future. Because of the unknown mergerringdown behavior in the case of binary neutron stars, which in any case gets removed from the waveform model, in practice only deviations $\delta \hat{\varphi}_{n}$ in the PN parameters $\varphi_{n}$ can be bounded. The set of possible testing parameters is taken to be

$\left\{\delta \hat{\varphi}_{-2}, \delta \hat{\varphi}_{0}, \delta \hat{\varphi}_{1}, \delta \hat{\varphi}_{2}, \delta \hat{\varphi}_{3}, \delta \hat{\varphi}_{4}, \delta \hat{\varphi}_{5}^{(\ell)}, \delta \hat{\varphi}_{6}, \delta \hat{\varphi}_{6}^{(\ell)}, \delta \hat{\varphi}_{7}\right\}$,

where the $\delta \hat{\varphi}_{n}$ are associated with powers of frequency $f^{(-5+n) / 3}$, and $\delta \hat{\varphi}_{5}^{(\ell)}$ and $\delta \hat{\varphi}_{6}^{(\ell)}$ with functions $\log (f)$ and $\log (f) f^{1 / 3}$, respectively; $\delta \hat{\varphi}_{5}$ would be completely degenerate with some reference phase $\phi_{c}$ and hence is not included in the list. In addition to corrections to the positive PN order coefficients, deviations at -1 PN are included because they offer the possibility to constrain the presence of dipole radiation during the inspiral (discussed below). $\delta \hat{\varphi}_{-2}$ and $\delta \hat{\varphi}_{1}$ represent absolute rather than relative deviations, as both are identically zero in GR. 

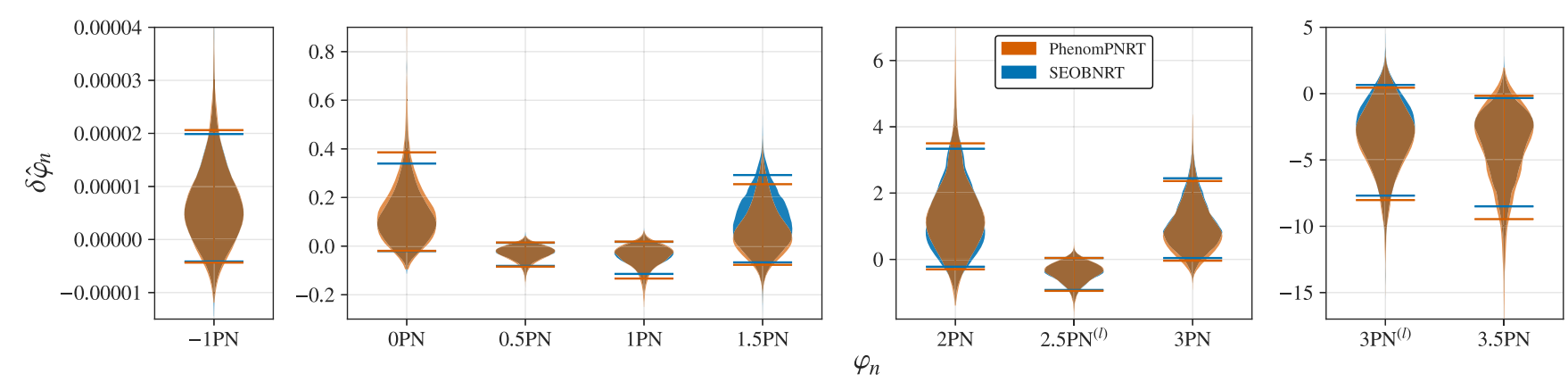

FIG. 1. Posterior density functions on deviations of PN coefficients $\delta \hat{\varphi}_{n}$ obtained using two different waveform models (PhenomPNRT and SEOBNRT); see the main text for details. The $-1 \mathrm{PN}$ and 0.5PN corrections correspond to absolute deviations, whereas all others represent fractional deviations from the PN coefficient in GR. The horizontal bars indicate $90 \%$ credible regions.

We also employ the SEOBNRV4 waveform model, which is constructed from an aligned-spin EOB model for binary black holes augmented with information from NR simulations [58]. Using the methods of Ref. [59], this model is evaluated in the frequency domain, and then we add the tidal correction $\phi_{T}(f)$ as described above; we refer to the resulting waveform model as SEOBNRT. Unlike PhenomPNRT, the SEOBNRT model is not constructed explicitly in terms of PN coefficients $\varphi_{n}$. Instead, we model the effect of a relative shift $\delta \hat{\varphi}_{n}$ by adding to the frequency domain phase a term $\delta \hat{\varphi}_{n} \varphi_{n} f^{(-5+n) / 3}$ or $\delta \hat{\varphi}_{n}^{(\ell)} \varphi_{n}^{(\ell)} f^{(-5+n) / 3} \log (f)$, as applicable. These corrections are then tapered to zero at the merger frequency.

Figure 1 depicts the PDFs on $\delta \hat{\varphi}_{n}$ recovered when only variations at that particular PN order are allowed. We find that the phase evolution of GW170817 is consistent with the GR prediction. The $90 \%$ credible region for each parameter contains the GR value of $\delta \hat{\varphi}_{n}=0$ at all orders other than 3PN and 3.5PN. [Using PhenomPNRT (SEOBNRT), the GR value lies at the 6.8th (4.4th) percentile of the PDF for the 3PN parameter and at the 95.0th (96.7th) percentile for the 3.5PN parameter.] For the pipeline used to perform parametrized tests with binary black holes, it has been shown in Ref. [28] through extensive simulations that no noticeable systematics are present. In the case of binary neutron stars such a study is computationally demanding because of the long signals, and a similar study will be published at a later date. At present we have no reason to believe that the offsets seen here at 3PN and 3.5PN have anything other than a statistical origin. In any case, we note that the value of zero is in the support of the posterior density function for all testing parameters. The bounds on the positive-PN parameters $(n \geq 0)$ obtained with GW170817 alone are comparable to those obtained by combining the binary black hole signals GW150914, GW151226, and GW170104 in Ref. [16] using the IMRPhenomPv2 waveform model. For convenience we also separately give $90 \%$ upper bounds on deviations in PN coefficients; see Fig. 2.

The PDFs shown in Fig. 1 were constructed using the same choice of prior distribution outlined in Ref. [19] with the following modifications. We use uniform priors on $\delta \hat{\varphi}_{n}$ that are broad enough to fully contain the plotted PDFs. Because of the degeneracy between $\delta \hat{\varphi}_{0}$ and the chirp mass, a broader prior distribution was chosen for the latter as compared to Ref. [19] for runs in which $\delta \hat{\varphi}_{0}$ was allowed to vary. All inference was done assuming the prior $\left|\chi_{i}\right| \leq 0.99$, where $\chi_{i}=c \mathbf{S}_{i} /\left(G m_{i}^{2}\right)$ is the dimensionless spin of each body. This conservative spin prior was chosen to allow the constraints on $\delta \hat{\varphi}_{n}$ to be directly compared with those from binary black hole observations, which used the same prior $[13,15]$. Nevertheless, throughout this Letter we assume the two objects to be neutron stars, and following Ref. [19] we limit our prior on the component tidal parameters to $\Lambda_{i} \leq 5000$. (For a precise definition of the $\Lambda_{i}$, see Ref. [1] and references therein.) This choice was motivated by reasonable astrophysical assumptions regarding the expected ranges for neutron star masses and equations of state [46,60,61]; higher values of $\Lambda$ are possible for some equations of state if the neutron star masses are small $\left(\simeq 0.9 M_{\odot}\right)$. The extra freedom introduced by including $\delta \hat{\varphi}_{n}$ leads to a loss in sensitivity in the measurement of tidal parameters; in particular, the tail of the PDF for the tidal deformation of the less massive body $\Lambda_{2}$ touches the prior

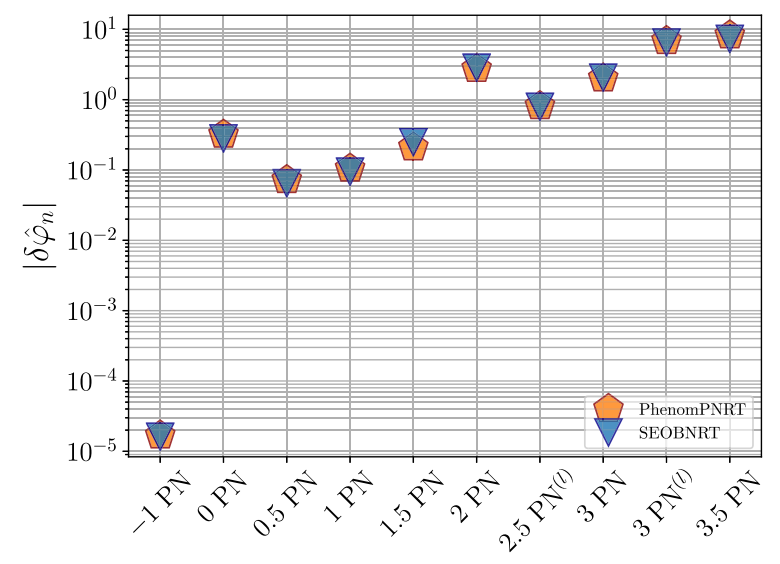

FIG. 2. $90 \%$ upper bounds on deviations $\left|\delta \hat{\varphi}_{n}\right|$ in the PN coefficients following from the posterior density functions shown in Fig. 1. 
upper bound in many of the tests. The correlation between $\delta \hat{\varphi}_{n}$ and $\Lambda_{2}$ means that the upper bounds for $\left|\delta \hat{\varphi}_{n}\right|$ would be weaker if we did not impose our neutron star prior of $\Lambda_{i} \leq 5000$.

Certain differences are present between the PhenomPNRT and SEOBNRT waveform models and the way they are used. First, PhenomPNRT allows for precessing spin configurations, whereas the SEOBNRT is restricted to systems with spins aligned with the orbital angular momentum. Second, continuity conditions enforced in the construction of PhenomPNRT waveforms cause deviations from GR in the inspiral to affect the behavior of later phases of the signal, whereas the tapering of deviations in SEOBNRT ensures that the merger-ringdown of the underlying waveform is exactly reproduced. However, this discrepancy is not expected to affect measurements of $\delta \hat{\varphi}_{n}$ significantly because the signal is dominated by the inspiral and both waveform models are amplitude tapered near merger. Third, the spin-induced quadrupole moment [62], which enters the phase at $2 \mathrm{PN}$ through quadrupole-monopole couplings, is computed using neutron-star universal relations [63] in PhenomPNRT and is assumed to take the black-hole value in SEOBNRT. Finally, in the PhenomPNRT model, fractional deviations are applied only to nonspinning terms in the PN expansion of the phase; i.e., terms dependent on the bodies' spins retain their GR values (There is no fundamental reason for this choice; we follow the convention used in previous publications on parametrized tests of GR $[13,15,16,27,64]$.). In SEOBNRT, fractional deviations are applied to all terms at a given post-Newtonian order. One can convert between these two parametrizations post hoc by requiring that the total phase correction be the same with either choice; the results shown in Figs. 1 and 2 correspond to the parametrization used by PhenomPNRT. Nevertheless, the different treatment of the spin terms may still explain the discrepancy seen at $1.5 \mathrm{PN}$, where spin effects first enter. (In the SEOBNRT parametrization, the $\mathrm{PDF}$ for $\delta \hat{\varphi}_{7}$ touches the prior bounds. After mapping to the PhenomPNRT parametrization, these tails of the distribution are down weighted, so our final results are a good approximation to the complete PDF.) Either parametrization offers a reasonable phenomenological description of deviations from GR; the generally close correspondence at most PN orders between results from the two models indicates that the quantities measured can be interpreted in similar ways. For more details on each waveform model we use, see Table I of Ref. [19].

The long inspiral observed in GW170817 (relative to previous binary black hole signals) allows us to place the first stringent constraints on $\delta \hat{\varphi}_{-2}$. This parameter is of particular interest due to its association with dipole radiation, i.e., radiation sourced by a time-varying dipole moment of the binary. Dipole radiation is forbidden in pure GR; however, adding other long-range fields to theory - either in the gravitational sector (e.g., massless scalar-tensor theories) or nongravitational sector (e.g., electromagnetism)—enables this new dissipative channel. The additional energy flux induces a negative $-1 \mathrm{PN}$ order correction to the phase evolution, provided that dipole radiation only contributes a small correction to the total flux predicted in GR. The precise nature of the additional long-range fields determines the dependence of this -1 PN correction on the various other parameters describing the binary (e.g., masses, spins, etc); in line with the theory-agnostic approach pursued here, we assume no a priori correlation between dipole radiation and the other binary parameters by using a uniform prior on $\delta \hat{\varphi}_{-2}$.

Writing the total energy flux as $\mathcal{F}_{\mathrm{GW}}=\mathcal{F}_{\mathrm{GR}}\left(1+B c^{2} / v^{2}\right)$, the leading-order modification to the phase due to theory-agnostic effects of dipole radiation is given by $\delta \hat{\varphi}_{-2}=-4 B / 7[65,66]$. Combining the PDFs shown in Fig. 1 obtained with the PhenomPNRT and SEOBNRT waveforms, converting to a PDF on $B$ using the previous relation, and restricting to the physical parameter space $B \geq 0$ corresponding to positive outgoing flux, the presence of dipole radiation in GW170817 can be constrained to $B \leq 1.2 \times 10^{-5}$. For comparison, precise timing of radio pulses from the double pulsar PSR J0737-3039 offers some of the best current theory-agnostic constraints $|B| \lesssim 10^{-7}$ [66-70]. (Neutron star-white dwarf binaries offer stronger constraints than the double pulsar on certain specific alternative theories of gravity [71-73], but provide comparable theory-agnostic constraints.) This much stronger constraint arises, in part, because of the much longer observation time over which the inspirals of binary pulsars are tracked.

Though our bound on the dipole parameter $B$ is weaker than existing constraints, it is the first that comes directly from the nonlinear and dynamical regime of gravity achieved during compact binary coalescences. In this regard, we note that for general scalar-tensor theories there are regions of parameter space where constraints from both Solar System and binary pulsar observations are satisfied, and yet new effects not present in GR appear in the frequency range of GW detectors, such as spontaneous scalarization $[74,75]$ or resonant excitation [76,77] of a massive field, or dynamical scalarization [72,78-81].

Constraints from gravitational wave propagation.-The propagation of GWs may differ in theories beyond GR, and the deviations depend on the distance that the GWs travel. The search for such deviations provides unique tests of relativity, particularly when the distance inferred through GWs can be compared with an accurate, independent distance measurement from EM observations. In GR, GWs propagate nondispersively at the speed of light with an amplitude inversely proportional to the distance traveled. Using GW170817, we carry out two different types of analyses to study the propagation of GWs, looking for possible deviations from GR's predictions. The first method implements a generic modification to the GW dispersion relation, adding terms that correct for a massive 
graviton, and momentum dependent dispersion that could be apparent in Lorentz violating models [82,83]. The second modifies the distance relation GWs follow in GR by adding correcting factors accounting for the GW's gravitational leakage into the large extra dimensions of higher-dimensional theories of gravity $[84,85]$.

In GR, gravitational waves propagate at the speed of light and are nondispersive, leading to a dispersion relation $E^{2}=p^{2} c^{2}$. An alternative theory may generically modify this as $E^{2}=p^{2} c^{2}+A p^{\alpha} c^{\alpha}$, where $A$ is the coefficient of modified dispersion corresponding to the exponent denoted by $\alpha[82,83]$. When $\alpha=0$, a modification with $A>0$ may be interpreted as due to a nonzero graviton mass $\left(A=m_{g}^{2} c^{4}\right)$ [83]. It can be shown that such modified dispersion relations would lead to corrections to the $\mathrm{GW}$ phasing, thereby allowing us to constrain any dispersion of GWs [83]. This method, implemented in a Bayesian framework, placed bounds on $A$ corresponding to different $\alpha$ using binary black hole detections [16]. We apply the above method to constrain dispersion of GWs in the case of the binary neutron star merger GW170817 [1]. We find that GW170817 places weaker bounds on dispersion of GWs than the binary black holes. For instance, the bound on the graviton mass $m_{g}$ we obtain from GW170817 is $9.51 \times$ $10^{-22} \mathrm{eV} / c^{2}$, which is weaker compared to the bounds reported in Ref. [16]. This is not surprising as GW170817 is the closest source detected so far, and for the same SNR propagation-based tests such as this are more effective when the sources are farther away. This method complements the bounds on nondispersive standard model extension coefficients [86] reported in Ref. [2] from GW170817.

In higher-dimensional theories of gravity the scaling between the GW strain and the luminosity distance of the source is expected to be modified, suggesting a damping of the waveform due to gravitational leakage into large extra dimensions. This deviation from the GR scaling $h_{\mathrm{GR}} \propto d_{L}^{-1}$ depends on the number of dimensions $D>4$ and would result in a systematic overestimation of the source luminosity distance inferred from GW observations $[84,85]$. A comparison of distance measurements from GW and EM observations of GW170817 allows us to constrain the presence of large additional spacetime dimensions. We assume, as is the case in many extra-dimensional models, that light and matter propagate in four spacetime dimensions only, thus allowing us to infer the EM luminosity distance $d_{L}^{\mathrm{EM}}$. In the absence of a complete, unique GW model in higher-dimensional gravity, we use a phenomenological ansatz for the GW amplitude scaling and neglect all other effects of modified gravity in the GW phase and amplitude. This approach requires that gravity be asymptotically GR in the strong-field regime, while modifications due to leakage into extra dimensions start to appear at large distances from the source. We therefore consider gravity modifications with a screening mechanism, i.e., a phenomenological model with a characteristic length scale $R_{c}$ beyond which the propagating GWs start to leak into higher dimensions. In this model, the strain scales as

$$
h \propto \frac{1}{d_{L}^{\mathrm{GW}}}=\frac{1}{d_{L}^{\mathrm{EM}}}\left[1+\left(\frac{d_{L}^{\mathrm{EM}}}{R_{c}}\right)^{n}\right]^{-(D-4) /(2 n)},
$$

where $D$ denotes the number of spacetime dimensions, and where $R_{c}$ and $n$ are the distance scale of the screening and the transition steepness, respectively. Equation (2) reduces to the standard GR scaling at distances much shorter than $R_{c}$, and the model is consistent with tests of GR performed in the Solar System or with binary pulsars. Unlike the scaling relation considered in Refs. [84,85], notice that Eq. (2) reduces to the GR limit for $D=4$ spacetime dimensions. An independent measurement of the source luminosity distance from EM observations of GW170817 allows us to infer the number of spacetime dimensions from a comparison of the GW and EM distance estimates, for given values of model parameters $R_{c}$ and $n$. Constraints on the number of spacetime dimensions are derived in a framework of Bayesian analysis, from the joint posterior probability for $D$, $d_{L}^{\mathrm{GW}}$, and $d_{L}^{\mathrm{EM}}$, given the two statistically independent measurements of EM data $x_{\mathrm{EM}}$ and GW data $x_{\mathrm{GW}}$. The posterior for $D$ is then given by

$$
\begin{aligned}
p\left(D \mid x_{\mathrm{GW}}, x_{\mathrm{EM}}\right)= & \int p\left(d_{L}^{\mathrm{GW}} \mid x_{\mathrm{GW}}\right) p\left(d_{L}^{\mathrm{EM}} \mid x_{\mathrm{EM}}\right) \\
& \times \delta\left[D-D\left(d_{L}^{\mathrm{GW}}, d_{L}^{\mathrm{EM}}, R_{c}, n\right)\right] d d_{L}^{\mathrm{GW}} d d_{L}^{\mathrm{EM}} .
\end{aligned}
$$

As in Ref. [19], we use a measurement of the surface brightness fluctuation distance to the host galaxy NGC 4993 from Ref. [87] to constrain the EM distance, assuming a Gaussian distribution for the posterior probability $p\left(d_{L}^{\mathrm{EM}} \mid x_{\mathrm{EM}}\right)$, with the mean value and standard deviation given by $40.7 \pm 2.4 \mathrm{Mpc}$ [87]. Contrary to Ref. [85], our analysis relies on a direct measurement of $d_{L}^{\mathrm{EM}}$ and is independent of prior information on $H_{0}$ or any other cosmological parameter. For the measurement of the GW distance, the posterior distribution $p\left(d_{L}^{\mathrm{GW}} \mid x_{\mathrm{GW}}\right)$ was inferred from the GW data assuming general relativity and fixing the sky position to the optical counterpart while marginalizing over all other waveform parameters [19]. Our analysis imposes a prior on the GW luminosity distance that is consistent with a four-dimensional universe, but we have checked that other reasonable prior choices do not significantly modify the results. We invert the scaling relation in Eq. (2) to compute $D\left(d_{L}^{\mathrm{GW}}, d_{L}^{\mathrm{EM}}, R_{c}, n\right)$ in Eq. (3). Figure 3 shows the $90 \%$ upper bounds on the number of dimensions $D$, for theories with a certain transition steepness $n$ and distance scale $R_{c}$. Shading indicates the excluded regions of parameter space. Our results are consistent with the GR prediction of $D=4$. 


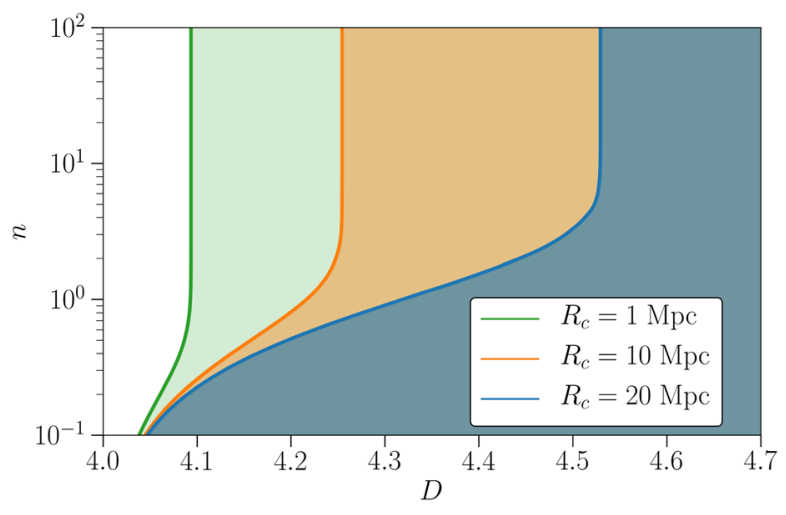

FIG. 3. $90 \%$ upper bounds on the number of spacetime dimensions $D$, assuming fixed transition steepness $n$ and distance scale $R_{c}$. Shading indicates the regions of parameter space excluded by the data.

Additionally, the data allow us to infer constraints on the characteristic distance scale $R_{c}$ of higher-dimensional theories with a screening mechanism, while fixing $D$ to 5,6 , or 7 . The posterior for $p\left(R_{c} \mid x_{\mathrm{GW}}, x_{\mathrm{EM}}\right)$ is obtained from the joint posterior probability of $R_{c}, d_{L}^{\mathrm{GW}}$, and $d_{L}^{\mathrm{EM}}$, given by a function that is formally the same as Eq. (3), but with $D$ and $R_{c}$ switching places. We fix the model parameters $D$ and $n$ and compute $R_{c}\left(d_{L}^{\mathrm{GW}}, d_{L}^{\mathrm{EM}}, D, n\right)$ by inverting the scaling relation in Eq. (2). Since we consider higher-dimensional models that allow only for a relative damping of the GW signal, we select posterior samples with $d_{L}^{\mathrm{GW}}>d_{L}^{\mathrm{EM}}$, leading to an additional step function $\theta\left(d_{L}^{\mathrm{GW}}-d_{L}^{\mathrm{EM}}\right)$ in $p\left(R_{c} \mid x_{\mathrm{GW}}, x_{\mathrm{EM}}\right)$. In Fig. 4, we show $10 \%$ lower bounds on the screening radius $R_{c}$, for theories with a certain fixed transition steepness $n$ and number of dimensions $D>4$. Shading indicates the excluded regions of parameter space. For higher-dimensional theories of gravity with a characteristic length scale $R_{c}$ of the order of the Hubble radius $R_{H} \sim 4 \mathrm{Gpc}$, such as the well-known

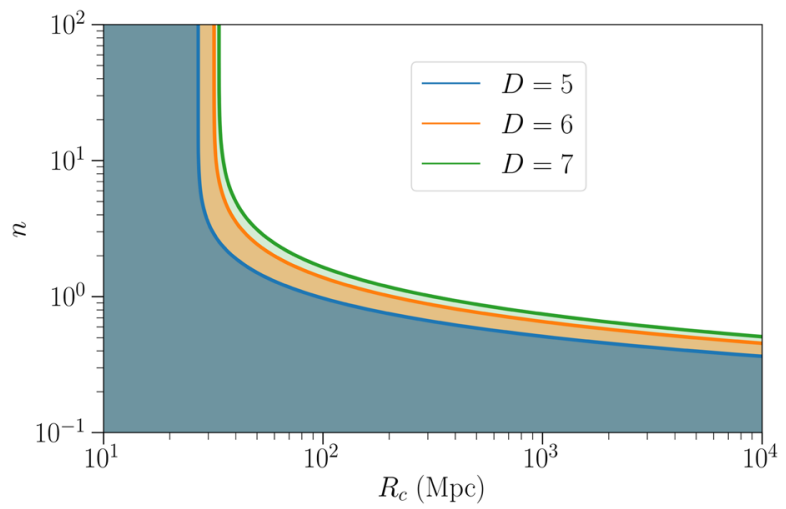

FIG. 4. $10 \%$ lower limits on the distance scale $R_{c}$ (in Mpc), assuming fixed transition steepness $n$ and number of spacetime dimensions $D$. Shading indicates the regions of parameter space excluded by the data.
Dvali-Gabadadze-Porrati (DGP) models of dark energy $[88,89]$, small transition steepnesses $[n \sim \mathcal{O}(0.1)]$ are excluded by the data. Our analysis cannot conclusively rule out DGP models that provide a sufficiently steep transition $(n>1)$ between GR and the onset of gravitational leakage. Future LIGO-Virgo observations of binary neutron star mergers, especially at higher redshifts, have the potential to place stronger constraints on higher-dimensional gravity.

Constraints on the polarization of gravitational waves.-Generic metric theories of gravity predict up to six polarization modes for metric perturbations: two tensor (helicity \pm 2 ), two vector (helicity \pm 1 ), and two scalar (helicity 0) modes [90,91]. GWs in GR, however, have only the two tensor modes regardless of the source properties; any detection of a nontensor mode would be an unambiguous indication of physics beyond GR. The GW strain measured by a detector can be written in general as $h(t)=F^{A} h_{A}$, where $h_{A}$ are the 6 independent polarization modes and $F^{A}$ represent the detector responses to the different modes $A=(+, \times, x, y, b, l)$. The antenna response functions depend only on the detector orientation and GW helicity; i.e., they are independent of the intrinsic properties of the source. We can therefore place bounds on the polarization content of GW170817 by studying which combination of response functions is consistent with the signal observed [92-96].

The first test on the polarization of GWs was performed for GW150914 [13]. The number of GR polarization modes expected was equal to the number of detectors in the network that observed GW150914, rendering this test inconclusive. The addition of Virgo to the network of GW detectors allowed for the first informative test of polarization for GW170814 [17]. This analysis established that the GW data were better described by pure tensor modes than pure vector or pure scalar modes, with Bayes factors in favor of tensor modes of more than 200 and 1000, respectively.

We here carry out a test similar to Ref. [17] by performing a coherent Bayesian analysis of the signal properties with the three interferometer outputs, using either the tensor or the vector or the scalar response functions. (Note that although the SNR in Virgo was significantly lower than in the two LIGO detectors, the Virgo data stream still carries information about the signal.) We assume that the phase evolution of the GW can be described by GR templates, but the polarization content can vary [97]. The phase evolution is modeled with the GR waveform model IMRPhenomPv2 and the analysis is carried out with LALInference [40]. Tidal effects are not included in this waveform model, but this is not expected to affect the results presented here, since the polarization test is sensitive to the antenna pattern functions of the detectors and not the phase evolution of the signal, as argued. The analysis described tests for the presence of pure tensor, vector, or scalar modes. We leave the analysis of mixed-mode content to future work. 
If the sky location of GW170817 is constrained to NGC 4993, we find overwhelming evidence in favor of pure tensor polarization modes in comparison to pure vector and pure scalar modes with a (base ten) logarithm of the Bayes factor of $+20.81 \pm 0.08$ and $+23.09 \pm 0.08$, respectively. This result is many orders of magnitude stronger than the GW170814 case both due to the sky position of GW170817 relative to the detectors and the fact that the sky position is determined precisely by electromagnetic observations. Indeed if the sky location is unconstrained we find evidence against scalar modes with $+5.84 \pm 0.09$, while the test is inconclusive for vector modes with $+0.72 \pm 0.09$.

Conclusions. - Using the binary neutron star coalescence signal GW170817, and in some cases also its associated electromagnetic counterpart, we have subjected general relativity to a range of tests related to the dynamics of the source (putting bounds on deviations of PN coefficients), the propagation of gravitational waves (constraining local Lorentz invariance violations, as well as large extra dimensions), and the polarization content of gravitational waves. In all cases we find agreement with the predictions of GR.

The upcoming observing runs of the LIGO and Virgo detectors are expected to result in more detections of binary neutron star coalescences [98]. Along with electromagnetic observations, combining information from gravitational wave events (including binary black hole mergers) will lead to increasingly more stringent constraints on deviations from general relativity $[27,28]$, or conceivably potential evidence of the theory's shortcomings.

The authors gratefully acknowledge the support of the United States National Science Foundation (NSF) for the construction and operation of the LIGO Laboratory and Advanced LIGO as well as the Science and Technology Facilities Council (STFC) of the United Kingdom, the Max-Planck-Society (MPS), and the State of Niedersachsen/Germany for support of the construction of Advanced LIGO and construction and operation of the GEO600 detector. Additional support for Advanced LIGO was provided by the Australian Research Council. The authors gratefully acknowledge the Italian Istituto Nazionale di Fisica Nucleare (INFN), the French Centre National de la Recherche Scientifique (CNRS), and the Foundation for Fundamental Research on Matter supported by the Netherlands Organisation for Scientific Research, for the construction and operation of the Virgo detector and the creation and support of the EGO consortium. The authors also gratefully acknowledge research support from these agencies as well as by the Council of Scientific and Industrial Research of India, the Department of Science and Technology, India, the Science \& Engineering Research Board (SERB), India, the Ministry of Human Resource Development, India, the Spanish Agencia Estatal de Investigación, the Vicepresidència i Conselleria
d'Innovació, Recerca i Turisme and the Conselleria d'Educació i Universitat del Govern de les Illes Balears, the Conselleria d'Educació, Investigació, Cultura i Esport de la Generalitat Valenciana, the National Science Centre of Poland, the Swiss National Science Foundation (SNSF), the Russian Foundation for Basic Research, the Russian Science Foundation, the European Commission, the European Regional Development Funds (ERDF), the Royal Society, the Scottish Funding Council, the Scottish Universities Physics Alliance, the Hungarian Scientific Research Fund (OTKA), the Lyon Institute of Origins (LIO), the Paris Île-de-France Region, the National Research, Development and Innovation Office Hungary (NKFIH), the National Research Foundation of Korea, Industry Canada and the Province of Ontario through the Ministry of Economic Development and Innovation, the Natural Science and Engineering Research Council Canada, the Canadian Institute for Advanced Research, the Brazilian Ministry of Science, Technology, Innovations, and Communications, the International Center for Theoretical Physics South American Institute for Fundamental Research (ICTP-SAIFR), the Research Grants Council of Hong Kong, the National Natural Science Foundation of China (NSFC), the Leverhulme Trust, the Research Corporation, the Ministry of Science and Technology (MOST), Taiwan and the Kavli Foundation. The authors gratefully acknowledge the support of the NSF, STFC, MPS, INFN, CNRS, and the State of Niedersachsen/Germany for provision of computational resources.

[1] B. Abbott et al. (Virgo and LIGO Scientific Collaborations), Phys. Rev. Lett. 119, 161101 (2017).

[2] B. P. Abbott et al. (Virgo, Fermi-GBM, INTEGRAL, and LIGO Scientific Collaborations), Astrophys. J. 848, L13 (2017).

[3] B. P. Abbott et al., Astrophys. J. 848, L12 (2017).

[4] T. Baker, E. Bellini, P. G. Ferreira, M. Lagos, J. Noller, and I. Sawicki, Phys. Rev. Lett. 119, 251301 (2017).

[5] P. Creminelli and F. Vernizzi, Phys. Rev. Lett. 119, 251302 (2017).

[6] J. Sakstein and B. Jain, Phys. Rev. Lett. 119, 251303 (2017).

[7] J. M. Ezquiaga and M. Zumalacárregui, Phys. Rev. Lett. 119, 251304 (2017).

[8] D. Langlois, R. Saito, D. Yamauchi, and K. Noui, Phys. Rev. D 97, 061501(R) (2018).

[9] A. Dima and F. Vernizzi, Phys. Rev. D 97, 101302(R) (2018).

[10] C. de Rham and S. Melville, Phys. Rev. Lett. 121, 221101 (2018).

[11] S. Boran, S. Desai, E. O. Kahya, and R. P. Woodard, Phys. Rev. D 97, 041501(R) (2018).

[12] B. P. Abbott et al. (Virgo and LIGO Scientific Collaborations), Phys. Rev. Lett. 116, 061102 (2016).

[13] B. P. Abbott et al. (Virgo and LIGO Scientific Collaborations), Phys. Rev. Lett. 116, 221101 (2016). 
[14] B. P. Abbott et al. (Virgo and LIGO Scientific Collaborations), Phys. Rev. Lett. 116, 241103 (2016).

[15] B. P. Abbott et al. (Virgo and LIGO Scientific Collaborations), Phys. Rev. X 6, 041015 (2016).

[16] B. P. Abbott et al. (Virgo and LIGO Scientific Collaborations), Phys. Rev. Lett. 118, 221101 (2017).

[17] B. P. Abbott et al. (Virgo and LIGO Scientific Collaborations), Phys. Rev. Lett. 119, 141101 (2017).

[18] B. P. Abbott et al. (Virgo and LIGO Scientific Collaborations), Astrophys. J. 851, L35 (2017).

[19] B. P. Abbott et al. (LIGO Scientific and Virgo Collaborations), Phys. Rev. X 9, 011001 (2019).

[20] B. P. Abbott et al. (LIGO Scientific and Virgo Collaborations), Phys. Rev. Lett. 121, 161101 (2018).

[21] J. R. Gair, M. Vallisneri, S. L. Larson, and J. G. Baker, Living Rev. Relativity 16, 7 (2013).

[22] N. Yunes and X. Siemens, Living Rev. Relativity 16, 9 (2013).

[23] L. Blanchet and B. S. Sathyaprakash, Phys. Rev. Lett. 74, 1067 (1995).

[24] K. G. Arun, B. R. Iyer, M. S. S. Qusailah, and B. S. Sathyaprakash, Phys. Rev. D 74, 024006 (2006).

[25] N. Yunes and F. Pretorius, Phys. Rev. D 80, 122003 (2009).

[26] C. K. Mishra, K. G. Arun, B. R. Iyer, and B. S. Sathyaprakash, Phys. Rev. D 82, 064010 (2010).

[27] T. G. F. Li, W. Del Pozzo, S. Vitale, C. Van Den Broeck, M. Agathos, J. Veitch, K. Grover, T. Sidery, R. Sturani, and A. Vecchio, Phys. Rev. D 85, 082003 (2012).

[28] J. Meidam et al., Phys. Rev. D 97, 044033 (2018).

[29] S. Husa, S. Khan, M. Hannam, M. Pürrer, F. Ohme, X. J. Forteza, and A. Bohé, Phys. Rev. D 93, 044006 (2016).

[30] S. Khan, S. Husa, M. Hannam, F. Ohme, M. Pürrer, X. J. Forteza, and A. Bohé, Phys. Rev. D 93, 044007 (2016).

[31] M. Hannam, P. Schmidt, A. Bohé, L. Haegel, S. Husa, F. Ohme, G. Pratten, and M. Pürrer, Phys. Rev. Lett. 113, 151101 (2014).

[32] L. Blanchet, T. Damour, B. R. Iyer, C. M. Will, and A. G. Wiseman, Phys. Rev. Lett. 74, 3515 (1995).

[33] L. Blanchet, T. Damour, G. Esposito-Farese, and B. R. Iyer, Phys. Rev. Lett. 93, 091101 (2004).

[34] L. E. Kidder, Phys. Rev. D 52, 821 (1995).

[35] L. Blanchet, A. Buonanno, and G. Faye, Phys. Rev. D 74, 104034 (2006); 81, 089901(E) (2010).

[36] L. Blanchet, A. Buonanno, and G. Faye, Phys. Rev. D 84, 064041 (2011).

[37] B. Mikoczi, M. Vasuth, and L. A. Gergely, Phys. Rev. D 71, 124043 (2005).

[38] A. Bohé, G. Faye, S. Marsat, and E. K. Porter, Classical Quantum Gravity 32, 195010 (2015).

[39] S. Marsat, Classical Quantum Gravity 32, 085008 (2015).

[40] J. Veitch et al., Phys. Rev. D 91, 042003 (2015).

[41] L. Sampson, N. Cornish, and N. Yunes, Phys. Rev. D 87, 102001 (2013).

[42] T. G. F. Li, W. Del Pozzo, S. Vitale, C. Van Den Broeck, M. Agathos, J. Veitch, K. Grover, T. Sidery, R. Sturani, and A. Vecchio, J. Phys. Conf. Ser. 363, 012028 (2012).

[43] N. Yunes, K. Yagi, and F. Pretorius, Phys. Rev. D 94, 084002 (2016).

[44] L. Blanchet, Living Rev. Relativity 17, 2 (2014).
[45] E. E. Flanagan and T. Hinderer, Phys. Rev. D 77, 021502(R) (2008).

[46] T. Hinderer, B. D. Lackey, R. N. Lang, and J. S. Read, Phys. Rev. D 81, 123016 (2010).

[47] J. Vines, E. E. Flanagan, and T. Hinderer, Phys. Rev. D 83, 084051 (2011).

[48] B. S. Sathyaprakash and S. V. Dhurandhar, Phys. Rev. D 44, 3819 (1991).

[49] A. Buonanno, B. R. Iyer, E. Ochsner, Y. Pan, and B. S. Sathyaprakash, Phys. Rev. D 80, 084043 (2009).

[50] K. G. Arun, A. Buonanno, G. Faye, and E. Ochsner, Phys. Rev. D 79, 104023 (2009); Phys. Rev. D 84, 049901(E) (2011).

[51] C. K. Mishra, A. Kela, K. G. Arun, and G. Faye, Phys. Rev. D 93, 084054 (2016).

[52] J. A. Faber and F. A. Rasio, Living Rev. Relativity 15, 8 (2012).

[53] S. Bernuzzi, A. Nagar, T. Dietrich, and T. Damour, Phys. Rev. Lett. 114, 161103 (2015).

[54] T. Hinderer et al., Phys. Rev. Lett. 116, 181101 (2016).

[55] T. Dietrich, S. Bernuzzi, and W. Tichy, Phys. Rev. D 96, 121501(R) (2017).

[56] T. Dietrich et al., Phys. Rev. D 99, 024029 (2019).

[57] P. Schmidt, M. Hannam, and S. Husa, Phys. Rev. D 86, 104063 (2012).

[58] A. Bohé et al., Phys. Rev. D 95, 044028 (2017).

[59] M. Pürrer, Classical Quantum Gravity 31, 195010 (2014).

[60] J. M. Lattimer, Annu. Rev. Nucl. Part. Sci. 62, 485 (2012).

[61] Y. Suwa, T. Yoshida, M. Shibata, H. Umeda, and K. Takahashi, Mon. Not. R. Astron. Soc. 481, 3305 (2018).

[62] E. Poisson, Phys. Rev. D 57, 5287 (1998).

[63] K. Yagi and N. Yunes, Science 341, 365 (2013).

[64] B. P. Abbott et al. (LIGO Scientific and Virgo Collaborations), arXiv:1903.04467.

[65] K. G. Arun, Classical Quantum Gravity 29, 075011 (2012).

[66] E. Barausse, N. Yunes, and K. Chamberlain, Phys. Rev. Lett. 116, 241104 (2016).

[67] A. G. Lyne et al., Science 303, 1153 (2004).

[68] M. Kramer et al., Science 314, 97 (2006).

[69] N. Yunes and S. A. Hughes, Phys. Rev. D 82, 082002 (2010).

[70] M. Kramer, Int. J. Mod. Phys. D 25, 1630029 (2016).

[71] P. C. C. Freire, N. Wex, G. Esposito-Farese, J. P. W. Verbiest, M. Bailes, B. A. Jacoby, M. Kramer, I. H. Stairs, J. Antoniadis, and G. H. Janssen, Mon. Not. R. Astron. Soc. 423, 3328 (2012).

[72] L. Shao, N. Sennett, A. Buonanno, M. Kramer, and N. Wex, Phys. Rev. X 7, 041025 (2017).

[73] D. Anderson, P. Freire, and N. Yunes, arXiv:1901.00938.

[74] T. Damour and G. Esposito-Farese, Phys. Rev. Lett. 70, 2220 (1993).

[75] F. M. Ramazanoğlu and F. Pretorius, Phys. Rev. D 93, 064005 (2016).

[76] V. Cardoso, S. Chakrabarti, P. Pani, E. Berti, and L. Gualtieri, Phys. Rev. Lett. 107, 241101 (2011).

[77] L. Sampson, N. Cornish, and N. Yunes, Phys. Rev. D 89, 064037 (2014).

[78] E. Barausse, C. Palenzuela, M. Ponce, and L. Lehner, Phys. Rev. D 87, 081506(R) (2013). 
[79] M. Shibata, K. Taniguchi, H. Okawa, and A. Buonanno, Phys. Rev. D 89, 084005 (2014).

[80] C. Palenzuela, E. Barausse, M. Ponce, and L. Lehner, Phys. Rev. D 89, 044024 (2014).

[81] L. Sampson, N. Yunes, N. Cornish, M. Ponce, E. Barausse, A. Klein, C. Palenzuela, and L. Lehner, Phys. Rev. D 90, 124091 (2014).

[82] C. M. Will, Phys. Rev. D 57, 2061 (1998).

[83] S. Mirshekari, N. Yunes, and C. M. Will, Phys. Rev. D 85, 024041 (2012).

[84] C. Deffayet and K. Menou, Astrophys. J. 668, L143 (2007).

[85] K. Pardo, M. Fishbach, D. E. Holz, and D. N. Spergel, J. Cosmol. Astropart. Phys. 07 (2018) 048.

[86] V. A. Kostelecky and N. Russell, Rev. Mod. Phys. 83, 11 (2011).

[87] M. Cantiello et al., Astrophys. J. 854, L31 (2018).

[88] G. R. Dvali, G. Gabadadze, and M. Porrati, Phys. Lett. B 485, 208 (2000).

[89] A. Lue, Phys. Rep. 423, 1 (2006).

[90] D. M. Eardley, D. L. Lee, and A. P. Lightman, Phys. Rev. D 8, 3308 (1973).
[91] C. M. Will, Living Rev. Relativity 17, 4 (2014).

[92] K. Chatziioannou, N. Yunes, and N. Cornish, Phys. Rev. D 86, 022004 (2012).

[93] M. Isi, M. Pitkin, and A. J. Weinstein, Phys. Rev. D 96, 042001 (2017).

[94] T. Callister, A. S. Biscoveanu, N. Christensen, M. Isi, A. Matas, O. Minazzoli, T. Regimbau, M. Sakellariadou, J. Tasson, and E. Thrane, Phys. Rev. X 7, 041058 (2017).

[95] B. P. Abbott et al. (Virgo and LIGO Scientific Collaborations), Phys. Rev. Lett. 120, 201102 (2018).

[96] B. P. Abbott et al. (Virgo and LIGO Scientific Collaborations), Phys. Rev. Lett. 120, 031104 (2018).

[97] M. Isi and A. J. Weinstein, arXiv:1710.03794.

[98] B. P. Abbott et al. (LIGO Scientific, Virgo, and KAGRA Collaborations), Living Rev. Relativity 21, 3 (2018).

Correction: Two authors (O. Bock and H.-B. Eggenstein) requested that their names be removed from the author list, which has been implemented.

B. P. Abbott, ${ }^{1}$ R. Abbott, ${ }^{1}$ T. D. Abbott, ${ }^{2}$ F. Acernese, ${ }^{3,4}$ K. Ackley, ${ }^{5}$ C. Adams, ${ }^{6}$ T. Adams, ${ }^{7}$ P. Addesso, ${ }^{8}$ R. X. Adhikari, ${ }^{1}$ V. B. Adya, ${ }^{9,10}$ C. Affeldt, ${ }^{9,10}$ B. Agarwal, ${ }^{11}$ M. Agathos, ${ }^{12}$ K. Agatsuma, ${ }^{13}$ N. Aggarwal, ${ }^{14}$ O. D. Aguiar, ${ }^{15}$ L. Aiello, ${ }^{16,17}$ A. Ain, ${ }^{18}$ P. Ajith, ${ }^{19}$ B. Allen,,${ }^{9,20,10}$ G. Allen, ${ }^{11}$ A. Allocca, ${ }^{21,22}$ M. A. Aloy, ${ }^{23}$ P. A. Altin, ${ }^{24}$ A. Amato, ${ }^{25}$ A. Ananyeva, S. B. Anderson, ${ }^{1}$ W. G. Anderson, ${ }^{20}$ S. V. Angelova, ${ }^{26}$ S. Antier, ${ }^{27}$ S. Appert, ${ }^{1}$ K. Arai, ${ }^{1}$ M. C. Araya, ${ }^{1}$ J. S. Areeda, ${ }^{28}$ M. Arène, ${ }^{29}$ N. Arnaud, ${ }^{27,30}$ K. G. Arun, ${ }^{31}$ S. Ascenzi, ${ }^{32,33}$ G. Ashton, ${ }^{5}$ M. Ast, ${ }^{34}$ S. M. Aston, ${ }^{6}$ P. Astone, ${ }^{35}$ D. V. Atallah, ${ }^{36}$ F. Aubin, ${ }^{7}$ P. Aufmuth, ${ }^{10}$ C. Aulbert, ${ }^{9}$ K. AultONeal, ${ }^{37}$ C. Austin, ${ }^{2}$ A. Avila-Alvarez, ${ }^{28}$ S. Babak, ${ }^{38,29}$ P. Bacon, ${ }^{29}$ F. Badaracco, ${ }^{16,17}$ M. K. M. Bader, ${ }^{13}$ S. Bae, ${ }^{39}$ P. T. Baker, ${ }^{40}$ F. Baldaccini, ${ }^{41,42}$ G. Ballardin, ${ }^{30}$ S. W. Ballmer, ${ }^{43}$ S. Banagiri, ${ }^{44}$ J. C. Barayoga, ${ }^{1}$ S. E. Barclay, ${ }^{45}$ B. C. Barish, ${ }^{1}$ D. Barker, ${ }^{46}$ K. Barkett, ${ }^{47}$ S. Barnum, ${ }^{14}$ F. Barone, ${ }^{3,4}$ B. Barr, ${ }^{45}$ L. Barsotti, ${ }^{14}$ M. Barsuglia, ${ }^{29}$ D. Barta, ${ }^{48}$ J. Bartlett, ${ }^{46}$ I. Bartos, ${ }^{49}$ R. Bassiri, ${ }^{50}$ A. Basti, ${ }^{21,22}$ J. C. Batch, ${ }^{46}$ M. Bawaj, ${ }^{51,42}$ J. C. Bayley, ${ }^{45}$ M. Bazzan, ${ }^{52,53}$ B. Bécsy, ${ }^{54}$ C. Beer, ${ }^{9}$ M. Bejger, ${ }^{55}$ I. Belahcene, ${ }^{27}$ A. S. Bell, ${ }^{45}$ D. Beniwal, ${ }^{56}$ M. Bensch, ${ }^{9,10}$ B. K. Berger, ${ }^{1}$ G. Bergmann, ${ }^{9,10}$ S. Bernuzzi, ${ }^{57,58}$ J. J. Bero, ${ }^{59}$ C. P. L. Berry, ${ }^{60}$ D. Bersanetti, ${ }^{61}$ A. Bertolini, ${ }^{13}$ J. Betzwieser, $^{6}$ R. Bhandare, ${ }^{62}$ I. A. Bilenko, ${ }^{63}$ S. A. Bilgili, ${ }^{40}$ G. Billingsley, ${ }^{1}$ C. R. Billman, ${ }^{49}$ J. Birch, ${ }^{6}$ R. Birney, ${ }^{26}$ O. Birnholtz, ${ }^{59}$ S. Biscans, ${ }^{1,14}$ S. Biscoveanu, ${ }^{5}$ A. Bisht, ${ }^{9,10}$ M. Bitossi, ${ }^{30,22}$ M. A. Bizouard, ${ }^{27}$ J. K. Blackburn, ${ }^{1}$ J. Blackman, ${ }^{47}$ C. D. Blair, ${ }^{6}$ D. G. Blair, ${ }^{64}$ R. M. Blair, ${ }^{46}$ S. Bloemen, ${ }^{65}$ N. Bode, ${ }^{9,10}$ M. Boer, ${ }^{66}$ Y. Boetzel, ${ }^{67}$ G. Bogaert, ${ }^{66}$ A. Bohe, ${ }^{38}$ F. Bondu, ${ }^{68}$ E. Bonilla, ${ }^{50}$ R. Bonnand, ${ }^{7}$ P. Booker, ${ }^{9,10}$ B. A. Boom, ${ }^{13}$ C. D. Booth, ${ }^{36}$ R. Bork, ${ }^{1}$ V. Boschi, ${ }^{30}$ S. Bose, ${ }^{69,18}$ K. Bossie, ${ }^{6}$ V. Bossilkov, ${ }^{64}$ J. Bosveld, ${ }^{64}$ Y. Bouffanais, ${ }^{29}$ A. Bozzi, ${ }^{30}$ C. Bradaschia, ${ }^{22}$ P. R. Brady, ${ }^{20}$ A. Bramley, ${ }^{6}$ M. Branchesi, ${ }^{16,17}$ J. E. Brau, ${ }^{70}$ T. Briant, ${ }^{71}$ F. Brighenti, ${ }^{72,73}$ A. Brillet, ${ }^{66}$ M. Brinkmann, ${ }^{9,10}$ V. Brisson, ${ }^{27, \dagger}$ P. Brockill, ${ }^{20}$ A. F. Brooks, ${ }^{1}$ D. D. Brown, ${ }^{56}$ S. Brunett, ${ }^{1}$ C. C. Buchanan, ${ }^{2}$ A. Buikema, ${ }^{14}$ T. Bulik, ${ }^{74}$ H. J. Bulten, ${ }^{75,13}$ A. Buonanno, ${ }^{38,76}$ D. Buskulic, ${ }^{7}$ C. Buy, ${ }^{29}$ R. L. Byer, ${ }^{50}$ M. Cabero, ${ }^{9}$ L. Cadonati, ${ }^{77}$ G. Cagnoli, ${ }^{25,78}$ C. Cahillane, ${ }^{1}$ J. Calderón Bustillo, ${ }^{77}$ T. A. Callister, ${ }^{1}$ E. Calloni, ${ }^{79,4}$ J. B. Camp, ${ }^{80}$ M. Canepa, ${ }^{81,61}$ P. Canizares, ${ }^{65}$ K. C. Cannon, ${ }^{82}$ H. Cao, ${ }^{56}$ J. Cao, ${ }^{83}$ C. D. Capano, ${ }^{9}$ E. Capocasa, ${ }^{29}$ F. Carbognani, ${ }^{30}$ S. Caride, ${ }^{84}$ M. F. Carney, ${ }^{85}$ G. Carullo, ${ }^{21}$ J. Casanueva Diaz, ${ }^{22}$ C. Casentini, ${ }^{32,33}$

S. Caudill, ${ }^{13,20}$ M. Cavaglià, ${ }^{86}$ F. Cavalier, ${ }^{27}$ R. Cavalieri, ${ }^{30}$ G. Cella, ${ }^{22}$ C. B. Cepeda, ${ }^{1}$ P. Cerdá-Durán, ${ }^{23}$ G. Cerretani, ${ }^{21,22}$ E. Cesarini, ${ }^{87,33}$ O. Chaibi, ${ }^{66}$ S. J. Chamberlin, ${ }^{88}$ M. Chan, ${ }^{45}$ S. Chao, ${ }^{89}$ P. Charlton, ${ }^{90}$ E. Chase, ${ }^{91}$ E. Chassande-Mottin, ${ }^{29}$ D. Chatterjee, ${ }^{20}$ K. Chatziioannou, ${ }^{92}$ B. D. Cheeseboro, ${ }^{40}$ H. Y. Chen, ${ }^{93}$ X. Chen, ${ }^{64}$ Y. Chen, ${ }^{47}$ H.-P. Cheng, ${ }^{49}$ H. Y. Chia, ${ }^{49}$ A. Chincarini, ${ }^{61}$ A. Chiummo, ${ }^{30}$ T. Chmiel, ${ }^{85}$ H. S. Cho, ${ }^{94}$ M. Cho, ${ }^{76}$ J. H. Chow, ${ }^{24}$ N. Christensen, ${ }^{95,66}$ Q. Chu, ${ }^{64}$ A. J. K. Chua, ${ }^{47}$ S. Chua, ${ }^{71}$ K. W. Chung, ${ }^{96}$ S. Chung, ${ }^{64}$ G. Ciani, ${ }^{52,53,49}$ A. A. Ciobanu, ${ }^{56}$ R. Ciolfi, ${ }^{97,98}$ F. Cipriano, ${ }^{66}$ C. E. Cirelli, ${ }^{50}$ A. Cirone, ${ }^{81,61}$ F. Clara, ${ }^{46}$ J. A. Clark, ${ }^{77}$ P. Clearwater, ${ }^{99}$ F. Cleva, ${ }^{66}$ C. Cocchieri, ${ }^{86}$ E. Coccia, ${ }^{16,17}$ P.-F. Cohadon, ${ }^{71}$ D. Cohen, ${ }^{27}$ A. Colla, ${ }^{100,35}$ C. G. Collette, ${ }^{101}$ C. Collins, ${ }^{60}$ L. R. Cominsky, ${ }^{102}$ M. Constancio Jr., ${ }^{15}$ L. Conti, ${ }^{53}$ S. J. Cooper, ${ }^{6}$ P. Corban, ${ }^{6}$ T. R. Corbitt, ${ }^{2}$ I. Cordero-Carrión, ${ }^{103}$ K. R. Corley, ${ }^{104}$ N. Cornish, ${ }^{105}$ A. Corsi, ${ }^{84}$ 
S. Cortese, ${ }^{30}$ C. A. Costa, ${ }^{15}$ R. Cotesta, ${ }^{38}$ M. W. Coughlin, ${ }^{1}$ S. B. Coughlin, ${ }^{36,91}$ J.-P. Coulon, ${ }^{66}$ S. T. Countryman, ${ }^{104}$ P. Couvares, ${ }^{1}$ P. B. Covas, ${ }^{106}$ E. E. Cowan, ${ }^{77}$ D. M. Coward, ${ }^{64}$ M. J. Cowart, ${ }^{6}$ D. C. Coyne, ${ }^{1}$ R. Coyne,${ }^{107}$

J. D. E. Creighton, ${ }^{20}$ T. D. Creighton, ${ }^{108}$ J. Cripe, ${ }^{2}$ S. G. Crowder, ${ }^{109}$ T. J. Cullen, ${ }^{2}$ A. Cumming, ${ }^{45}$ L. Cunningham, ${ }^{45}$

E. Cuoco ${ }^{30}$ T. Dal Canton, ${ }^{80}$ G. Dálya, ${ }^{54}$ S. L. Danilishin,,${ }^{109}$ S. D’Antonio, ${ }^{33}$ K. Danzmann,,${ }^{910}$ A. Dasgupta, ${ }^{110}$

C. F. Da Silva Costa, ${ }^{49}$ V. Dattilo, ${ }^{30}$ I. Dave, ${ }^{62}$ M. Davier, ${ }^{27}$ D. Davis,${ }^{43}$ E. J. Daw, ${ }^{111}$ B. Day, ${ }^{77}$ D. DeBra, ${ }^{50}$

M. Deenadayalan, ${ }^{18}$ J. Degallaix, ${ }^{25}$ M. De Laurentis,${ }^{79,4}$ S. Deléglise, ${ }^{71}$ W. Del Pozzo, ${ }^{21,22}$ N. Demos, ${ }^{14}$ T. Denker, ${ }^{9,10}$ T. Dent, ${ }^{9}$ R. De Pietri, ${ }^{57,58}$ J. Derby, ${ }^{28}$ V. Dergachev,${ }^{9}$ R. De Rosa,${ }^{79,4}$ C. De Rossi ${ }^{25,30}$ R. DeSalvo, ${ }^{112}$ O. de Varona,${ }^{9,10}$ S. Dhurandhar, ${ }^{18}$ M. C. Díaz, ${ }^{108}$ T. Dietrich, ${ }^{13}$ L. Di Fiore, ${ }^{4}$ M. Di Giovanni, ${ }^{113,98}$ T. Di Girolamo, ${ }^{79,4}$ A. Di Lieto, ${ }^{21,22}$ B. Ding, ${ }^{101}$ S. Di Pace, ${ }^{100,35}$ I. Di Palma,,${ }^{100,35}$ F. Di Renzo, ${ }^{21,22}$ A. Dmitriev, ${ }^{60}$ Z. Doctor, ${ }^{93}$ V. Dolique, ${ }^{25}$ F. Donovan, ${ }^{14}$ K. L. Dooley, ${ }^{36,86}$ S. Doravari, ${ }^{9,10}$ I. Dorrington, ${ }^{36}$ M. Dovale Álvarez, ${ }^{60}$ T. P. Downes, ${ }^{20}$ M. Drago, ${ }^{9,16,17}$

C. Dreissigacker, ${ }^{9,10}$ J. C. Driggers, ${ }^{46}$ Z. Du, ${ }^{83}$ P. Dupej, ${ }^{45}$ S. E. Dwyer, ${ }^{46}$ P. J. Easter, ${ }^{5}$ T. B. Edo, ${ }^{111}$ M. C. Edwards, ${ }^{95}$ A. Effler, ${ }^{6}$ P. Ehrens, ${ }^{1}$ J. Eichholz, ${ }^{1}$ S. S. Eikenberry ${ }^{49}$ M. Eisenmann, ${ }^{7}$ R. A. Eisenstein, ${ }^{14}$ R. C. Essick, ${ }^{93}$ H. Estelles, ${ }^{106}$ D. Estevez, ${ }^{7}$ Z. B. Etienne, ${ }^{40}$ T. Etzel, ${ }^{1}$ M. Evans, ${ }^{14}$ T. M. Evans, ${ }^{6}$ V. Fafone,${ }^{32,33,16}$ H. Fair, ${ }^{43}$ S. Fairhurst, ${ }^{36}$ X. Fan,${ }^{83}$ S. Farinon, ${ }^{61}$ B. Farr, ${ }^{70}$ W. M. Farr, ${ }^{60}$ E. J. Fauchon-Jones,${ }^{36}$ M. Favata, ${ }^{114}$ M. Fays,${ }^{36}$ C. Fee, ${ }^{85}$ H. Fehrmann, ${ }^{9}$ J. Feicht, ${ }^{1}$ M. M. Fejer, ${ }^{50}$ F. Feng, ${ }^{29}$ A. Fernandez-Galiana, ${ }^{14}$ I. Ferrante, ${ }^{21,22}$ E. C. Ferreira, ${ }^{15}$ F. Ferrini, ${ }^{30}$ F. Fidecaro, ${ }^{21,22}$ I. Fiori, ${ }^{30}$ D. Fiorucci, ${ }^{29}$ M. Fishbach, ${ }^{93}$ R. P. Fisher, ${ }^{43}$ J. M. Fishner, ${ }^{14}$ M. Fitz-Axen ${ }^{44}$ R. Flaminio, ${ }^{7,115}$ M. Fletcher, ${ }^{45}$ H. Fong,${ }^{92}$ J. A. Font, ${ }^{23,116}$ P. W. F. Forsyth, ${ }^{24}$ S. S. Forsyth, ${ }^{77}$ J.-D. Fournier, ${ }^{66}$ S. Frasca, ${ }^{100,35}$ F. Frasconi, ${ }^{22}$ Z. Frei, ${ }^{54}$ A. Freise, ${ }^{60}$ R. Frey ${ }^{70}$ V. Frey ${ }^{27}$ P. Fritschel,${ }^{14}$ V. V. Frolov, ${ }^{6}$ P. Fulda, ${ }^{49}$ M. Fyffe, ${ }^{6}$ H. A. Gabbard, ${ }^{45}$ B. U. Gadre, ${ }^{18}$ S. M. Gaebel,${ }^{60}$ J. R. Gair, ${ }^{117}$ L. Gammaitoni, ${ }^{41}$ M. R. Ganija,${ }^{56}$ S. G. Gaonkar, ${ }^{18}$ A. Garcia, ${ }^{28}$ C. García-Quirós, ${ }^{106}$ F. Garufi, ${ }^{79,4}$ B. Gateley, ${ }^{46}$ S. Gaudio ${ }^{37}$ G. Gaur, ${ }^{118}$ V. Gayathri, ${ }^{119}$ G. Gemme, ${ }^{61}$ E. Genin, ${ }^{30}$ A. Gennai, ${ }^{22}$ D. George, ${ }^{11}$ J. George, ${ }^{62}$ L. Gergely, ${ }^{120}$ V. Germain, ${ }^{7}$ S. Ghonge, ${ }^{77}$ Abhirup Ghosh, ${ }^{19}$ Archisman Ghosh, ${ }^{13}$ S. Ghosh,${ }^{20}$ B. Giacomazzo, ${ }^{113,98}$ J. A. Giaime, ${ }^{2,6}$

K. D. Giardina, ${ }^{6}$ A. Giazotto, ${ }^{22, \dagger}$ K. Gill, ${ }^{37}$ G. Giordano, ${ }^{3,4}$ L. Glover, ${ }^{112}$ E. Goetz, ${ }^{46}$ R. Goetz, ${ }^{49}$ B. Goncharov, ${ }^{5}$ G. González, ${ }^{2}$ J. M. Gonzalez Castro, ${ }^{21,22}$ A. Gopakumar, ${ }^{121}$ M. L. Gorodetsky, ${ }^{63}$ S. E. Gossan, ${ }^{1}$ M. Gosselin, ${ }^{30}$ R. Gouaty ${ }^{7}$ A. Grado, ${ }^{122,4}$ C. Graef, ${ }^{45}$ M. Granata, ${ }^{25}$ A. Grant, ${ }^{45}$ S. Gras, ${ }^{14}$ C. Gray, ${ }^{46}$ G. Greco, ${ }^{72,73}$ A. C. Green, ${ }^{60}$ R. Green, ${ }^{36}$ E. M. Gretarsson, ${ }^{37}$ P. Groot, ${ }^{65}$ H. Grote, ${ }^{36}$ S. Grunewald, ${ }^{38}$ P. Gruning, ${ }^{27}$ G. M. Guidi, ${ }^{72,73}$ H. K. Gulati, ${ }^{110}$ X. Guo, ${ }^{83}$ A. Gupta, ${ }^{88}$ M. K. Gupta, ${ }^{110}$ K. E. Gushwa, ${ }^{1}$ E. K. Gustafson, ${ }^{1}$ R. Gustafson, ${ }^{123}$ O. Halim, ${ }^{17,16}$ B. R. Hall, ${ }^{69}$ E. D. Hall, ${ }^{14}$

E. Z. Hamilton, ${ }^{36}$ H. F. Hamilton, ${ }^{124}$ G. Hammond,${ }^{45}$ M. Haney,${ }^{67}$ M. M. Hanke, ${ }^{9,10}$ J. Hanks, ${ }^{46}$ C. Hanna, ${ }^{88}$

M. D. Hannam, ${ }^{36}$ O. A. Hannuksela, ${ }^{96}$ J. Hanson, ${ }^{6}$ T. Hardwick, ${ }^{2}$ J. Harms,${ }^{16,17}$ G. M. Harry, ${ }^{125}$ I. W. Harry, ${ }^{38}$ M. J. Hart, ${ }^{45}$ C.-J. Haster, ${ }^{92}$ K. Haughian, ${ }^{45}$ J. Healy, ${ }^{59}$ A. Heidmann, ${ }^{71}$ M. C. Heintze,${ }^{6}$ H. Heitmann, ${ }^{66}$ P. Hello, ${ }^{27}$ G. Hemming,${ }^{30}$ M. Hendry, ${ }^{45}$ I. S. Heng, ${ }^{45}$ J. Hennig, ${ }^{45}$ A. W. Heptonstall, ${ }^{1}$ F. J. Hernandez,${ }^{5}$ M. Heurs, ${ }^{9,10}$ S. Hild, ${ }^{45}$ T. Hinderer, ${ }^{65}$ D. Hoak, ${ }^{30}$ S. Hochheim, ${ }^{9,10}$ D. Hofman, ${ }^{25}$ N. A. Holland ${ }^{24}$ K. Holt, ${ }^{6}$ D. E. Holz,${ }^{93}$ P. Hopkins, ${ }^{36}$ C. Horst, ${ }^{20}$ J. Hough, ${ }^{45}$ E. A. Houston, ${ }^{45}$ E. J. Howell ${ }^{64}$ A. Hreibi, ${ }^{66}$ E. A. Huerta, ${ }^{11}$ D. Huet,${ }^{27}$ B. Hughey,${ }^{37}$ M. Hulko, ${ }^{1}$ S. Husa, ${ }^{106}$ S. H. Huttner,${ }^{45}$ T. Huynh-Dinh, ${ }^{6}$ A. Iess, ${ }^{32,33}$ N. Indik, ${ }^{9}$ C. Ingram, ${ }^{56}$ R. Inta, ${ }^{84}$ G. Intini, ${ }^{100,35}$ H. N. Isa, ${ }^{45}$ J.-M. Isac, ${ }^{71}$ M. Isi, ${ }^{1}$ B. R. Iyer, ${ }^{19}$ K. Izumi, ${ }^{46}$ T. Jacqmin, ${ }^{71}$ K. Jani, ${ }^{77}$ P. Jaranowski, ${ }^{126}$ D. S. Johnson, ${ }^{11}$ W. W. Johnson, ${ }^{2}$ D. I. Jones, ${ }^{127}$ R. Jones, ${ }^{45}$ R. J. G. Jonker, ${ }^{13}$ L. Ju, ${ }^{64}$ J. Junker, ${ }^{9,10}$ C. V. Kalaghatgi, ${ }^{36}$ V. Kalogera, ${ }^{91}$ B. Kamai, ${ }^{1}$ S. Kandhasamy, ${ }^{6}$ G. Kang ${ }^{39}$

J. B. Kanner, ${ }^{1}$ S. J. Kapadia, ${ }^{20}$ S. Karki,${ }^{70}$ K. S. Karvinen, ${ }^{9,10}$ M. Kasprzack, ${ }^{2}$ M. Katolik, ${ }^{11}$ S. Katsanevas, ${ }^{30}$

E. Katsavounidis, ${ }^{14}$ W. Katzman, ${ }^{6}$ S. Kaufer, ${ }^{9}{ }^{10}$ K. Kawabe, ${ }^{46}$ N. V. Keerthana, ${ }^{18}$ F. Kéfélian, ${ }^{66}$ D. Keitel, ${ }^{45}$ A. J. Kemball, ${ }^{11}$ R. Kennedy, ${ }^{111}$ J. S. Key, ${ }^{128}$ F. Y. Khalili, ${ }^{63}$ B. Khamesra, ${ }^{77}$ H. Khan, ${ }^{28}$ I. Khan, ${ }^{16,33}$ S. Khan, ${ }^{9}$ Z. Khan, ${ }^{110}$ E. A. Khazanov, ${ }^{129}$ N. Kijbunchoo, ${ }^{24}$ Chunglee Kim ${ }^{130}$ J. C. Kim, ${ }^{131}$ K. Kim, ${ }^{96}$ W. Kim, ${ }^{56}$ W. S. Kim, ${ }^{132}$ Y.-M. Kim, ${ }^{133}$ E. J. King, ${ }^{56}$ P. J. King, ${ }^{46}$ M. Kinley-Hanlon, ${ }^{125}$ R. Kirchhoff, ${ }^{9,10}$ J. S. Kissel, ${ }^{46}$ L. Kleybolte, ${ }^{34}$ S. Klimenko, ${ }^{49}$ T. D. Knowles, ${ }^{40}$ P. Koch,${ }^{9,10}$ S. M. Koehlenbeck, ${ }^{9,10}$ S. Koley ${ }^{13}$ V. Kondrashov, ${ }^{1}$ A. Kontos, ${ }^{14}$ M. Korobko, ${ }^{34}$ W. Z. Korth, ${ }^{1}$ I. Kowalska, ${ }^{74}$ D. B. Kozak, ${ }^{1}$ C. Krämer, ${ }^{9}$ V. Kringel,,${ }^{9,10}$ B. Krishnan, ${ }^{9}$ A. Królak, ${ }^{134,135}$ G. Kuehn, ${ }^{9,10}$ P. Kumar, ${ }^{136}$ R. Kumar, ${ }^{110}$ S. Kumar, ${ }^{19}$ L. Kuo, ${ }^{89}$ A. Kutynia, ${ }^{134}$ S. Kwang, ${ }^{20}$ B. D. Lackey, ${ }^{38}$ K. H. Lai, ${ }^{96}$ M. Landry, ${ }^{46}$ R. N. Lang, ${ }^{137}$ J. Lange, ${ }^{59}$ B. Lantz, ${ }^{50}$ R. K. Lanza, ${ }^{14}$ A. Lartaux-Vollard, ${ }^{27}$ P. D. Lasky, ${ }^{5}$ M. Laxen, ${ }^{6}$ A. Lazzarini, ${ }^{1}$ C. Lazzaro, ${ }^{53}$ P. Leaci, ${ }^{100,35}$ S. Leavey, ${ }^{9,10}$ C. H. Lee, ${ }^{94}$ H. K. Lee, ${ }^{138}$ H. M. Lee, ${ }^{130}$ H. W. Lee, ${ }^{131}$ K. Lee, ${ }^{45}$ J. Lehmann,,${ }^{910}$ A. Lenon, ${ }^{40}$ M. Leonardi, ${ }^{9} 10,115$ N. Leroy, ${ }^{27}$ N. Letendre, ${ }^{7}$ Y. Levin, ${ }^{5}$ J. Li,${ }^{83}$ T. G. F. Li, ${ }^{96}$ X. Li,${ }^{47}$ S. D. Linker, ${ }^{112}$ T. B. Littenberg, ${ }^{139}$ J. Liu, ${ }^{64}$ X. Liu, ${ }^{20}$ R. K. L. Lo, ${ }^{96}$ N. A. Lockerbie, ${ }^{26}$ L. T. London, ${ }^{36}$ A. Longo, ${ }^{140,141}$ M. Lorenzini, ${ }^{16,17}$ V. Loriette, ${ }^{142}$ M. Lormand, ${ }^{6}$ G. Losurdo, ${ }^{22}$ J. D. Lough,,${ }^{96}$ C. O. Lousto, ${ }^{59}$ G. Lovelace, ${ }^{28}$ H. Lück, ${ }^{9,10}$ D. Lumaca, ${ }^{32,33}$ A. P. Lundgren, ${ }^{9}$ R. Lynch, ${ }^{14}$ Y. Ma, ${ }^{47}$ R. Macas, ${ }^{36}$ S. Macfoy, ${ }^{26}$ B. Machenschalk, ${ }^{9}$ M. MacInnis, ${ }^{14}$ D. M. Macleod,${ }^{36}$ 
I. Magaña Hernandez,${ }^{20}$ F. Magaña-Sandoval,${ }^{43}$ L. Magaña Zertuche,${ }^{86}$ R. M. Magee, ${ }^{88}$ E. Majorana, ${ }^{35}$ I. Maksimovic, ${ }^{142}$ N. Man,${ }^{66}$ V. Mandic, ${ }^{44}$ V. Mangano, ${ }^{45}$ G. L. Mansell, ${ }^{24}$ M. Manske ${ }^{20,24}$ M. Mantovani, ${ }^{30}$ F. Marchesoni,${ }^{51,42}$ F. Marion, ${ }^{7}$ S. Márka, ${ }^{104}$ Z. Márka, ${ }^{104}$ C. Markakis, ${ }^{11}$ A. S. Markosyan,${ }^{50}$ A. Markowitz, ${ }^{1}$ E. Maros, ${ }^{1}$ A. Marquina, ${ }^{103}$ S. Marsat,${ }^{38}$ F. Martelli, ${ }^{72,73}$ L. Martellini, ${ }^{66}$ I. W. Martin, ${ }^{45}$ R. M. Martin,${ }^{114}$ D. V. Martynov, ${ }^{14}$ K. Mason, ${ }^{14}$ E. Massera, ${ }^{111}$ A. Masserot, ${ }^{7}$ T. J. Massinger, ${ }^{1}$ M. Masso-Reid, ${ }^{45}$ S. Mastrogiovanni, ${ }^{100,35}$ A. Matas ${ }^{44}$ F. Matichard, ${ }^{1,14}$ L. Matone,${ }^{104}$ N. Mavalvala, ${ }^{14}$ N. Mazumder, ${ }^{69}$ J. J. McCann, ${ }^{64}$ R. McCarthy, ${ }^{46}$ D. E. McClelland, ${ }^{24}$ S. McCormick, ${ }^{6}$ L. McCuller, ${ }^{14}$ S. C. McGuire, ${ }^{143}$ J. McIver, ${ }^{1}$ D. J. McManus, ${ }^{24}$ T. McRae, ${ }^{24}$ S. T. McWilliams, ${ }^{40}$ D. Meacher, ${ }^{88}$ G. D. Meadors, ${ }^{5}$ M. Mehmet, ${ }^{9,10}$ J. Meidam,${ }^{13}$ E. Mejuto-Villa, ${ }^{8}$ A. Melatos, ${ }^{99}$ G. Mendell, ${ }^{46}$ D. Mendoza-Gandara, ${ }^{9,10}$ R. A. Mercer, ${ }^{20}$ L. Mereni, ${ }^{25}$ E. L. Merilh, ${ }^{46}$ M. Merzougui ${ }^{66}$ S. Meshkov, ${ }^{1}$ C. Messenger, ${ }^{45}$ C. Messick, ${ }^{88}$ R. Metzdorff, ${ }^{71}$ P. M. Meyers, ${ }^{44}$ H. Miao, ${ }^{60}$ C. Michel, ${ }^{25}$ H. Middleton, ${ }^{99}$ E. E. Mikhailov, ${ }^{144}$ L. Milano, ${ }^{79,4}$ A. L. Miller, ${ }^{49}$ A. Miller, ${ }^{100,35}$ B. B. Miller, ${ }^{91}$ J. Miller, ${ }^{14}$ M. Millhouse, ${ }^{105}$ J. Mills, ${ }^{36}$ M. C. Milovich-Goff, ${ }^{112}$ O. Minazzoli, ${ }^{66,145}$ Y. Minenkov, ${ }^{33}$ J. Ming, ${ }^{9,10}$ C. Mishra, ${ }^{146}$ S. Mitra, ${ }^{18}$

V. P. Mitrofanov, ${ }^{63}$ G. Mitselmakher, ${ }^{49}$ R. Mittleman, ${ }^{14}$ D. Moffa ${ }^{85}$ K. Mogushi ${ }^{86}$ M. Mohan, ${ }^{30}$ S. R. P. Mohapatra, ${ }^{14}$ M. Montani, ${ }^{72,73}$ C. J. Moore, ${ }^{12}$ D. Moraru, ${ }^{46}$ G. Moreno, ${ }^{46}$ S. Morisaki, ${ }^{82}$ B. Mours, ${ }^{7}$ C. M. Mow-Lowry, ${ }^{60}$ G. Mueller, ${ }^{49}$ A. W. Muir, ${ }^{36}$ Arunava Mukherjee, ${ }^{9,10}$ D. Mukherjee, ${ }^{20}$ S. Mukherjee, ${ }^{108}$ N. Mukund, ${ }^{18}$ A. Mullavey, ${ }^{6}$ J. Munch, ${ }^{56}$ E. A. Muñiz,${ }^{43}$ M. Muratore, ${ }^{37}$ P. G. Murray, ${ }^{45}$ A. Nagar,${ }^{87,147,148}$ K. Napier,${ }^{77}$ I. Nardecchia, ${ }^{32,33}$ L. Naticchioni, ${ }^{100,35}$ R. K. Nayak ${ }^{149}$ J. Neilson, ${ }^{112}$ G. Nelemans, ${ }^{65,13}$ T. J. N. Nelson, ${ }^{6}$ M. Nery, ${ }^{9,10}$ A. Neunzert, ${ }^{123}$ L. Nevin, ${ }^{1}$ J. M. Newport, ${ }^{125}$ K. Y. Ng, ${ }^{14}$ S. Ng ${ }^{56}$ P. Nguyen, ${ }^{70}$ T. T. Nguyen, ${ }^{24}$ D. Nichols, ${ }^{65}$ A. B. Nielsen, ${ }^{9}$ S. Nissanke, ${ }^{65,13}$ A. Nitz, ${ }^{9}$ F. Nocera ${ }^{30}$ D. Nolting, ${ }^{6}$ C. North, ${ }^{36}$ L. K. Nuttall, ${ }^{36}$ M. Obergaulinger, ${ }^{23}$ J. Oberling, ${ }^{46}$ B. D. O'Brien, ${ }^{49}$ G. D. O' Dea, ${ }^{112}$ G. H. Ogin, ${ }^{150}$ J. J. Oh, ${ }^{132}$ S. H. Oh, ${ }^{132}$ F. Ohme, ${ }^{9}$ H. Ohta ${ }^{82}$ M. A. Okada, ${ }^{15}$ M. Oliver, ${ }^{106}$ P. Oppermann, ${ }^{9,10}$ Richard J. Oram, ${ }^{6}$ B. O’Reilly, ${ }^{6}$ R. Ormiston, ${ }^{44}$ L. F. Ortega, ${ }^{49}$ R. O'Shaughnessy, ${ }^{59}$ S. Ossokine, ${ }^{38}$ D. J. Ottaway, ${ }^{56}$ H. Overmier, ${ }^{6}$ B. J. Owen,${ }^{84}$ A. E. Pace, ${ }^{88}$ G. Pagano, ${ }^{21,22}$ J. Page, ${ }^{139}$ M. A. Page,${ }^{64}$ A. Pai,${ }^{119}$ S. A. Pai, ${ }^{62}$ J. R. Palamos,${ }^{70}$ O. Palashov, ${ }^{129}$ C. Palomba, ${ }^{35}$ A. Pal-Singh,${ }^{34}$ Howard Pan, ${ }^{89}$ Huang-Wei Pan, ${ }^{89}$ B. Pang, ${ }^{47}$ P. T. H. Pang, ${ }^{96}$ C. Pankow, ${ }^{91}$ F. Pannarale, ${ }^{36}$ B. C. Pant,${ }^{62}$ F. Paoletti, ${ }^{22}$ A. Paoli, ${ }^{30}$ M. A. Papa,${ }^{9,20,10}$ A. Parida ${ }^{18}$ W. Parker ${ }^{6}$ D. Pascucci, ${ }^{45}$ A. Pasqualetti, ${ }^{30}$ R. Passaquieti, ${ }^{21,22}$ D. Passuello, ${ }^{22}$ M. Patil, ${ }^{135}$ B. Patricelli, ${ }^{151,22}$ B. L. Pearlstone,${ }^{45}$ C. Pedersen,${ }^{36}$ M. Pedraza, ${ }^{1}$ R. Pedurand, ${ }^{25,152}$ L. Pekowsky, ${ }^{43}$ A. Pele, ${ }^{6}$ S. Penn, ${ }^{153}$ C. J. Perez, ${ }^{46}$ A. Perreca, ${ }^{113,98}$ L. M. Perri, ${ }^{91}$ H. P. Pfeiffer, ${ }^{92,38}$ M. Phelps, ${ }^{45}$ K. S. Phukon, ${ }^{18}$ O. J. Piccinni, ${ }^{100,35}$ M. Pichot, ${ }^{66}$ F. Piergiovanni, ${ }^{72,73}$ V. Pierro, ${ }^{8}$ G. Pillant,${ }^{30}$ L. Pinard, ${ }^{25}$ I. M. Pinto, ${ }^{8}$ M. Pirello, ${ }^{46}$ M. Pitkin, ${ }^{45}$ R. Poggiani, ${ }^{21,22}$ P. Popolizio, ${ }^{30}$ E. K. Porter, ${ }^{29}$ L. Possenti, ${ }^{154,73}$ A. Post, ${ }^{9}$ J. Powell, ${ }^{155}$ J. Prasad, ${ }^{18}$ J. W. W. Pratt, ${ }^{37}$ G. Pratten, ${ }^{106}$ V. Predoi, ${ }^{36}$ T. Prestegard, ${ }^{20}$ M. Principe,${ }^{8}$ S. Privitera, ${ }^{38}$ G. A. Prodi, ${ }^{113,98}$ L. G. Prokhorov, ${ }^{63}$ O. Puncken,,${ }^{9,10}$ M. Punturo, ${ }^{42}$ P. Puppo, ${ }^{35}$ M. Pürrer, ${ }^{38}$ H. Qi,${ }^{20}$ V. Quetschke, ${ }^{108}$ E. A. Quintero, ${ }^{1}$ R. Quitzow-James, ${ }^{70}$ F. J. Raab, ${ }^{46}$ D. S. Rabeling, ${ }^{24}$ H. Radkins, ${ }^{46}$ P. Raffai, ${ }^{54}$ S. Raja, ${ }^{62}$ C. Rajan, ${ }^{62}$ B. Rajbhandari, ${ }^{84}$ M. Rakhmanov, ${ }^{108}$ K. E. Ramirez, ${ }^{108}$ A. Ramos-Buades, ${ }^{106}$ Javed Rana, ${ }^{18}$ P. Rapagnani, ${ }^{100,35}$ V. Raymond, ${ }^{36}$ M. Razzano, ${ }^{21,22}$ J. Read, ${ }^{28}$ T. Regimbau, ${ }^{66,7}$ L. Rei, ${ }^{61}$ S. Reid,${ }^{26}$ D. H. Reitze, ${ }^{1,49}$ W. Ren, ${ }^{11}$ F. Ricci, ${ }^{100,35}$ P. M. Ricker, ${ }^{11}$ G. M. Riemenschneider, ${ }^{147,156}$ K. Riles, ${ }^{123}$ M. Rizzo,${ }^{59}$ N. A. Robertson, ${ }^{1,45}$ R. Robie, ${ }^{45}$ F. Robinet, ${ }^{27}$ T. Robson, ${ }^{105}$ A. Rocchi, ${ }^{33}$ L. Rolland, ${ }^{7}$ J. G. Rollins, ${ }^{1}$ V. J. Roma ${ }^{70}$ R. Romano, ${ }^{3,4}$ C. L. Romel, ${ }^{46}$ J. H. Romie, ${ }^{6}$ D. Rosińska, ${ }^{157,55}$ M. P. Ross, ${ }^{158}$ S. Rowan, ${ }^{45}$ A. Rüdiger, ${ }^{9,10}$ P. Ruggi, ${ }^{30}$ G. Rutins, ${ }^{159}$ K. Ryan,${ }^{46}$ S. Sachdev, ${ }^{1}$ T. Sadecki, ${ }^{46}$ M. Sakellariadou, ${ }^{160}$ L. Salconi, ${ }^{30}$ M. Saleem, ${ }^{119}$ F. Salemi, ${ }^{9}$ A. Samajdar, ${ }^{149,13}$ L. Sammut,,${ }^{5}$ L. M. Sampson, ${ }^{91}$ E. J. Sanchez, ${ }^{1}$ L. E. Sanchez, ${ }^{1}$ N. Sanchis-Gual, ${ }^{23}$ V. Sandberg, ${ }^{46}$ J. R. Sanders, ${ }^{43}$ N. Sarin, ${ }^{5}$ B. Sassolas, ${ }^{25}$ B. S. Sathyaprakash, ${ }^{88,36}$ P. R. Saulson, ${ }^{43}$ O. Sauter, ${ }^{123}$ R. L. Savage, ${ }^{46}$ A. Sawadsky, ${ }^{34}$ P. Schale,${ }^{70}$ M. Scheel, ${ }^{47}$ J. Scheuer,${ }^{91}$ P. Schmidt, ${ }^{65}$ R. Schnabel, ${ }^{34}$ R. M. S. Schofield, ${ }^{70}$ A. Schönbeck, ${ }^{34}$ E. Schreiber, ${ }^{9,10}$ D. Schuette, ${ }^{9,10}$ B. W. Schulte, ${ }^{9,10}$ B. F. Schutz, ${ }^{36,9}$ S. G. Schwalbe, ${ }^{37}$ J. Scott, ${ }^{45}$ S. M. Scott, ${ }^{24}$ E. Seidel, ${ }^{11}$ D. Sellers, ${ }^{6}$ A. S. Sengupta, ${ }^{161}$ N. Sennett, ${ }^{38}$

D. Sentenac, ${ }^{30}$ V. Sequino, ${ }^{32,33,16}$ A. Sergeev, ${ }^{129}$ Y. Setyawati, ${ }^{9}$ D. A. Shaddock, ${ }^{24}$ T. J. Shaffer, ${ }^{46}$ A. A. Shah, ${ }^{139}$ M. S. Shahriar, ${ }^{91}$ M. B. Shaner, ${ }^{12}$ L. Shao, ${ }^{38}$ B. Shapiro,${ }^{50}$ P. Shawhan, ${ }^{76}$ H. Shen, ${ }^{11}$ D. H. Shoemaker, ${ }^{14}$

D. M. Shoemaker ${ }^{77}$ K. Siellez,${ }^{77}$ X. Siemens, ${ }^{20}$ M. Sieniawska, ${ }^{55}$ D. Sigg ${ }^{46}$ A. D. Silva,${ }^{15}$ L. P. Singer, ${ }^{80}$ A. Singh,${ }^{9,10}$ A. Singhal, ${ }^{16,35}$ A. M. Sintes, ${ }^{106}$ B. J. J. Slagmolen, ${ }^{24}$ T. J. Slaven-Blair, ${ }^{64}$ B. Smith, ${ }^{6}$ J. R. Smith, ${ }^{28}$ R. J. E. Smith, ${ }^{5}$ S. Somala, ${ }^{162}$ E. J. Son, ${ }^{132}$ B. Sorazu, ${ }^{45}$ F. Sorrentino, ${ }^{61}$ T. Souradeep, ${ }^{18}$ A. P. Spencer, ${ }^{45}$ A. K. Srivastava, ${ }^{110}$ K. Staats,${ }^{37}$ D. A. Steer, ${ }^{29}$ M. Steinke, ${ }^{9,10}$ J. Steinlechner, ${ }^{34,45}$ S. Steinlechner, ${ }^{34}$ D. Steinmeyer, ${ }^{9,10}$ B. Steltner, ${ }^{9,10}$ S. P. Stevenson, ${ }^{155}$ D. Stocks, ${ }^{50}$ R. Stone, ${ }^{108}$ D. J. Stops,${ }^{60}$ K. A. Strain, ${ }^{45}$ G. Stratta, ${ }^{72,73}$ S. E. Strigin, ${ }^{63}$ A. Strunk, ${ }^{46}$ R. Sturani, ${ }^{163}$ A. L. Stuver, ${ }^{164}$ T. Z. Summerscales, ${ }^{165}$ L. Sun, ${ }^{99}$ S. Sunil, ${ }^{110}$ J. Suresh, ${ }^{18}$ P. J. Sutton, ${ }^{36}$ B. L. Swinkels, ${ }^{13}$ M. J. Szczepańczyk, ${ }^{37}$ M. Tacca, ${ }^{13}$ S. C. Tait, ${ }^{45}$ C. Talbot, ${ }^{5}$ D. Talukder, ${ }^{70}$ N. Tamanini,${ }^{38}$ D. B. Tanner, ${ }^{49}$ M. Tápai, ${ }^{120}$ 
A. Taracchini, ${ }^{38}$ J. D. Tasson, ${ }^{95}$ J. A. Taylor, ${ }^{139}$ R. Taylor, ${ }^{1}$ S. V. Tewari, ${ }^{153}$ T. Theeg, ${ }^{9,10}$ F. Thies,${ }^{9,10}$ E. G. Thomas, ${ }^{60}$ M. Thomas, ${ }^{6}$ P. Thomas, ${ }^{46}$ K. A. Thorne,${ }^{6}$ E. Thrane, ${ }^{5}$ S. Tiwari, ${ }^{16,98}$ V. Tiwari, ${ }^{36}$ K. V. Tokmakov, ${ }^{26} \mathrm{~K}$. Toland, ${ }^{45}$ M. Tonelli, ${ }^{21,22}$ Z. Tornasi, ${ }^{45}$ A. Torres-Forné, ${ }^{23}$ C. I. Torrie, ${ }^{1}$ D. Töyrä, ${ }^{60}$ F. Travasso, ${ }^{30,42}$ G. Traylor, ${ }^{6}$ J. Trinastic, ${ }^{49}$ M. C. Tringali, ${ }^{113,98}$ L. Trozzo, ${ }^{166,22}$ K. W. Tsang, ${ }^{13}$ M. Tse,${ }^{14}$ R. Tso, ${ }^{47}$ L. Tsukada, ${ }^{82}$ D. Tsuna,${ }^{82}$ D. Tuyenbayev, ${ }^{108}$ K. Ueno, ${ }^{20}$ D. Ugolini, ${ }^{167}$ A. L. Urban, ${ }^{1}$ S. A. Usman, ${ }^{36}$ H. Vahlbruch, ${ }^{9,10}$ G. Vajente, ${ }^{1}$ G. Valdes, ${ }^{2}$ N. van Bakel, ${ }^{13}$ M. van Beuzekom, ${ }^{13}$ J. F. J. van den Brand, ${ }^{75,13}$ C. Van Den Broeck, ${ }^{13,168}$ D. C. Vander-Hyde, ${ }^{43}$ L. van der Schaaf, ${ }^{13}$ J. V. van Heijningen, ${ }^{13}$ A. A. van Veggel, ${ }^{45}$ M. Vardaro, ${ }^{52,53}$ V. Varma,${ }^{47}$ S. Vass, ${ }^{1}$ M. Vasúth, ${ }^{48}$ A. Vecchio, ${ }^{60}$ G. Vedovato, ${ }^{53}$ J. Veitch, ${ }^{45}$ P. J. Veitch, ${ }^{56}$ K. Venkateswara, ${ }^{158}$ G. Venugopalan, ${ }^{1}$ D. Verkindt, ${ }^{7}$ F. Vetrano, ${ }^{72,73}$ A. Viceré, ${ }^{72,73}$ A. D. Viets, ${ }^{20}$ S. Vinciguerra, ${ }^{60}$ D. J. Vine, ${ }^{159}$ J.-Y. Vinet, ${ }^{66}$ S. Vitale, ${ }^{14}$ T. Vo, ${ }^{43}$ H. Vocca, ${ }^{41,42}$ C. Vorvick, ${ }^{46}$ S. P. Vyatchanin, ${ }^{63}$ A. R. Wade, ${ }^{1}$ L. E. Wade, ${ }^{85}$ M. Wade,${ }^{85}$ R. Walet, ${ }^{13}$ M. Walker, ${ }^{28}$ L. Wallace, ${ }^{1}$ S. Walsh,${ }^{20,9}$ G. Wang, ${ }^{16,22}$ H. Wang, ${ }^{60}$ J. Z. Wang, ${ }^{123}$ W. H. Wang, ${ }^{108}$ Y. F. Wang, ${ }^{96}$ R. L. Ward, ${ }^{24}$ J. Warner ${ }^{46}$ M. Was, ${ }^{7}$ J. Watchi, ${ }^{101}$ B. Weaver, ${ }^{46}$ L.-W. Wei, ${ }^{9,10}$ M. Weinert, ${ }^{9,10}$ A. J. Weinstein, ${ }^{1}$ R. Weiss,${ }^{14}$ F. Wellmann, ${ }^{9,10}$ L. Wen, ${ }^{64}$ E. K. Wessel,,${ }^{11}$ P. Weßels,${ }^{9,10}$ J. Westerweck, ${ }^{9}$ K. Wette, ${ }^{24}$ J. T. Whelan,${ }^{59}$ B. F. Whiting, ${ }^{49}$ C. Whittle, ${ }^{14}$ D. Wilken, ${ }^{9,10}$ D. Williams, ${ }^{45}$ R. D. Williams, ${ }^{1}$ A. R. Williamson, ${ }^{59,65}$ J. L. Willis, ${ }^{1,124}$ B. Willke, ${ }^{9,10}$ M. H. Wimmer, ${ }^{9,10}$ W. Winkler, ${ }^{9,10}$ C. C. Wipf, ${ }^{1}$ H. Wittel, ${ }^{9,10}$ G. Woan, ${ }^{45}$ J. Woehler, ${ }^{9,10}$ J. K. Wofford, ${ }^{59}$ W. K. Wong, ${ }^{96}$ J. Worden, ${ }^{46}$ J. L. Wright, ${ }^{45}$ D. S. Wu, ${ }^{9,10}$ D. M. Wysocki, ${ }^{59}$ S. Xiao, ${ }^{1}$ W. Yam, ${ }^{14}$ H. Yamamoto, ${ }^{1}$ C. C. Yancey ${ }^{76}$ L. Yang, ${ }^{169}$ M. J. Yap,${ }^{24}$ M. Yazback,,${ }^{49}$ Hang Yu, ${ }^{14}$ Haocun Yu, ${ }^{14}$ M. Yvert, ${ }^{7}$ A. Zadrożny, ${ }^{134}$ M. Zanolin, ${ }^{37}$ T. Zelenova, ${ }^{30}$ J.-P. Zendri, ${ }^{53}$ M. Zevin, ${ }^{91}$ J. Zhang, ${ }^{64}$ L. Zhang, ${ }^{1}$ M. Zhang, ${ }^{144}$ T. Zhang, ${ }^{45}$ Y.-H. Zhang, ${ }^{9,10}$ C. Zhao, ${ }^{64}$ M. Zhou, ${ }^{91}$ Z. Zhou, ${ }^{91}$ S. J. Zhu, ${ }^{9,10}$ X. J. Zhu, ${ }^{5}$ A. B. Zimmerman, ${ }^{170,92}$ M. E. Zucker, ${ }^{1,14}$ and J. Zweizig ${ }^{1}$

(LIGO Scientific Collaboration and Virgo Collaboration)

${ }^{1}$ LIGO, California Institute of Technology, Pasadena, California 91125, USA

${ }^{2}$ Louisiana State University, Baton Rouge, Louisiana 70803, USA

${ }^{3}$ Università di Salerno, Fisciano, I-84084 Salerno, Italy

${ }^{4}$ INFN, Sezione di Napoli, Complesso Universitario di Monte S. Angelo, I-80126 Napoli, Italy

${ }^{5}$ OzGrav, School of Physics \& Astronomy, Monash University, Clayton 3800, Victoria, Australia

${ }^{6}$ LIGO Livingston Observatory, Livingston, Louisiana 70754, USA

${ }^{7}$ Laboratoire d'Annecy de Physique des Particules (LAPP), Univ. Grenoble Alpes, Université Savoie Mont Blanc, CNRS/IN2P3, F-74941 Annecy, France

${ }^{8}$ University of Sannio at Benevento, I-82100 Benevento, Italy and INFN, Sezione di Napoli, I-80100 Napoli, Italy

${ }^{9}$ Max Planck Institute for Gravitational Physics (Albert Einstein Institute), D-30167 Hannover, Germany

${ }^{10}$ Leibniz Universität Hannover, D-30167 Hannover, Germany

${ }^{11}$ NCSA, University of Illinois at Urbana-Champaign, Urbana, Illinois 61801, USA

${ }^{12}$ University of Cambridge, Cambridge CB2 1TN, United Kingdom

${ }^{13}$ Nikhef, Science Park 105, 1098 XG Amsterdam, Netherlands

${ }^{14}$ LIGO, Massachusetts Institute of Technology, Cambridge, Massachusetts 02139, USA

${ }^{15}$ Instituto Nacional de Pesquisas Espaciais, 12227-010 São José dos Campos, São Paulo, Brazil

${ }^{16}$ Gran Sasso Science Institute (GSSI), I-67100 L'Aquila, Italy

${ }^{17}$ INFN, Laboratori Nazionali del Gran Sasso, I-67100 Assergi, Italy

${ }^{18}$ Inter-University Centre for Astronomy and Astrophysics, Pune 411007, India

${ }^{19}$ International Centre for Theoretical Sciences, Tata Institute of Fundamental Research, Bengaluru 560089, India

${ }^{20}$ University of Wisconsin-Milwaukee, Milwaukee, Wisconsin 53201, USA

${ }^{21}$ Università di Pisa, I-56127 Pisa, Italy

${ }^{22}$ INFN, Sezione di Pisa, I-56127 Pisa, Italy

${ }^{23}$ Departamento de Astronomía y Astrofísica, Universitat de València, E-46100 Burjassot, València, Spain

${ }^{24}$ OzGrav, Australian National University, Canberra, Australian Capital Territory 0200, Australia

${ }^{25}$ Laboratoire des Matériaux Avancés (LMA), CNRS/IN2P3, F-69622 Villeurbanne, France

${ }^{26}$ SUPA, University of Strathclyde, Glasgow G1 1XQ, United Kingdom

${ }^{27}$ LAL, Univ. Paris-Sud, CNRS/IN2P3, Université Paris-Saclay, F-91898 Orsay, France

${ }^{28}$ California State University Fullerton, Fullerton, California 92831, USA

${ }^{29}$ APC, AstroParticule et Cosmologie, Université Paris Diderot, CNRS/IN2P3, CEA/Irfu, Observatoire de Paris, Sorbonne Paris Cité, F-75205 Paris Cedex 13, France

${ }^{30}$ European Gravitational Observatory (EGO), I-56021 Cascina, Pisa, Italy 
${ }^{31}$ Chennai Mathematical Institute, Chennai 603103, India

${ }^{32}$ Università di Roma Tor Vergata, I-00133 Roma, Italy

${ }^{33}$ INFN, Sezione di Roma Tor Vergata, I-00133 Roma, Italy

${ }^{34}$ Universität Hamburg, D-22761 Hamburg, Germany

${ }^{35}$ INFN, Sezione di Roma, I-00185 Roma, Italy

${ }^{36}$ Cardiff University, Cardiff CF24 3AA, United Kingdom

${ }^{37}$ Embry-Riddle Aeronautical University, Prescott, Arizona 86301, USA

${ }^{38}$ Max Planck Institute for Gravitational Physics (Albert Einstein Institute), D-14476 Potsdam-Golm, Germany

${ }^{39}$ Korea Institute of Science and Technology Information, Daejeon 34141, Korea

${ }^{40}$ West Virginia University, Morgantown, West Virginia 26506, USA

${ }^{41}$ Università di Perugia, I-06123 Perugia, Italy

${ }^{42}$ INFN, Sezione di Perugia, I-06123 Perugia, Italy

${ }^{43}$ Syracuse University, Syracuse, New York 13244, USA

${ }^{44}$ University of Minnesota, Minneapolis, Minnesota 55455, USA

${ }^{45}$ SUPA, University of Glasgow, Glasgow G12 8QQ, United Kingdom

${ }^{46}$ LIGO Hanford Observatory, Richland, Washington 99352, USA

${ }^{47}$ Caltech CaRT, Pasadena, California 91125, USA

${ }^{48}$ Wigner RCP, RMKI, H-1121 Budapest, Konkoly Thege Miklós út 29-33, Hungary

${ }^{49}$ University of Florida, Gainesville, Florida 32611, USA

${ }^{50}$ Stanford University, Stanford, California 94305, USA

${ }^{51}$ Università di Camerino, Dipartimento di Fisica, I-62032 Camerino, Italy

${ }^{52}$ Università di Padova, Dipartimento di Fisica e Astronomia, I-35131 Padova, Italy

${ }^{53}$ INFN, Sezione di Padova, I-35131 Padova, Italy

${ }^{54}$ MTA-ELTE Astrophysics Research Group, Institute of Physics, Eötvös University, Budapest 1117, Hungary

${ }^{55}$ Nicolaus Copernicus Astronomical Center, Polish Academy of Sciences, 00-716, Warsaw, Poland

${ }^{56} \mathrm{OzGrav}$, University of Adelaide, Adelaide, South Australia 5005, Australia

${ }^{57}$ Dipartimento di Scienze Matematiche, Fisiche e Informatiche, Università di Parma, I-43124 Parma, Italy

${ }^{58}$ INFN, Sezione di Milano Bicocca, Gruppo Collegato di Parma, I-43124 Parma, Italy

${ }^{59}$ Rochester Institute of Technology, Rochester, New York 14623, USA

${ }^{60}$ University of Birmingham, Birmingham B15 2TT, United Kingdom

${ }^{61}$ INFN, Sezione di Genova, I-16146 Genova, Italy

${ }^{62}$ RRCAT, Indore, Madhya Pradesh 452013, India

${ }^{63}$ Faculty of Physics, Lomonosov Moscow State University, Moscow 119991, Russia

${ }^{64}$ OzGrav, University of Western Australia, Crawley, Western Australia 6009, Australia

${ }^{65}$ Department of Astrophysics/IMAPP, Radboud University Nijmegen, P.O. Box 9010, 6500 GL Nijmegen, Netherlands

${ }^{66}$ Artemis, Université Côte d'Azur, Observatoire Côte d'Azur, CNRS, CS 34229, F-06304 Nice Cedex 4, France

${ }^{67}$ Physik-Institut, University of Zurich, Winterthurerstrasse 190, 8057 Zurich, Switzerland

${ }^{68}$ Univ Rennes, CNRS, Institut FOTON-UMR6082, F-3500 Rennes, France

${ }^{69}$ Washington State University, Pullman, Washington 99164, USA

${ }^{70}$ University of Oregon, Eugene, Oregon 97403, USA

${ }^{71}$ Laboratoire Kastler Brossel, Sorbonne Université, CNRS, ENS-Université PSL, Collège de France, F-75005 Paris, France

${ }^{72}$ Università degli Studi di Urbino 'Carlo Bo', I-61029 Urbino, Italy

${ }^{73}$ INFN, Sezione di Firenze, I-50019 Sesto Fiorentino, Firenze, Italy

${ }^{74}$ Astronomical Observatory Warsaw University, 00-478 Warsaw, Poland

${ }^{75}$ VU University Amsterdam, 1081 HV Amsterdam, Netherlands

${ }^{76}$ University of Maryland, College Park, Maryland 20742, USA

${ }^{77}$ School of Physics, Georgia Institute of Technology, Atlanta, Georgia 30332, USA

${ }^{78}$ Université Claude Bernard Lyon 1, F-69622 Villeurbanne, France

${ }^{79}$ Università di Napoli 'Federico II', Complesso Universitario di Monte S.Angelo, I-80126 Napoli, Italy

${ }^{80}$ NASA Goddard Space Flight Center, Greenbelt, Maryland 20771, USA

${ }^{81}$ Dipartimento di Fisica, Università degli Studi di Genova, I-16146 Genova, Italy

${ }^{82}$ RESCEU, University of Tokyo, Tokyo, 113-0033, Japan

${ }^{83}$ Tsinghua University, Beijing 100084, China

${ }^{84}$ Texas Tech University, Lubbock, Texas 79409, USA

${ }^{85}$ Kenyon College, Gambier, Ohio 43022, USA

${ }^{86}$ The University of Mississippi, University, Mississippi 38677, USA

${ }^{87}$ Museo Storico della Fisica e Centro Studi e Ricerche "Enrico Fermi", I-00184 Roma, Italyrico Fermi, I-00184 Roma, Italy

${ }^{88}$ The Pennsylvania State University, University Park, Pennsylvania 16802, USA

${ }^{89}$ National Tsing Hua University, Hsinchu City, 30013 Taiwan, Republic of China

${ }^{90}$ Charles Sturt University, Wagga Wagga, New South Wales 2678, Australia 
${ }^{91}$ Center for Interdisciplinary Exploration \& Research in Astrophysics (CIERA), Northwestern University, Evanston, Illinois 60208, USA

${ }^{92}$ Canadian Institute for Theoretical Astrophysics, University of Toronto, Toronto, Ontario M5S 3H8, Canada

${ }^{93}$ University of Chicago, Chicago, Illinois 60637, USA

${ }^{94}$ Pusan National University, Busan 46241, Korea

${ }^{95}$ Carleton College, Northfield, Minnesota 55057, USA

${ }^{96}$ The Chinese University of Hong Kong, Shatin, NT, Hong Kong

${ }^{97}$ INAF, Osservatorio Astronomico di Padova, I-35122 Padova, Italy

${ }^{98}$ INFN, Trento Institute for Fundamental Physics and Applications, I-38123 Povo, Trento, Italy

${ }^{99}$ OzGrav, University of Melbourne, Parkville, Victoria 3010, Australia

${ }^{100}$ Università di Roma 'La Sapienza', I-00185 Roma, Italy

${ }^{101}$ Université Libre de Bruxelles, Brussels 1050, Belgium

${ }^{102}$ Sonoma State University, Rohnert Park, California 94928, USA

${ }^{103}$ Departamento de Matemáticas, Universitat de València, E-46100 Burjassot, València, Spain

${ }^{104}$ Columbia University, New York, New York 10027, USA

${ }^{105}$ Montana State University, Bozeman, Montana 59717, USA

${ }^{106}$ Universitat de les Illes Balears, IAC3-IEEC, E-07122 Palma de Mallorca, Spain

${ }^{107}$ University of Rhode Island

${ }^{108}$ The University of Texas Rio Grande Valley, Brownsville, Texas 78520, USA

${ }^{109}$ Bellevue College, Bellevue, Washington 98007, USA

${ }^{110}$ Institute for Plasma Research, Bhat, Gandhinagar 382428, India

${ }^{111}$ The University of Sheffield, Sheffield S10 2TN, United Kingdom

${ }^{112}$ California State University, Los Angeles, 5151 State University Dr, Los Angeles, California 90032, USA

${ }^{113}$ Università di Trento, Dipartimento di Fisica, I-38123 Povo, Trento, Italy

${ }^{114}$ Montclair State University, Montclair, New Jersey 07043, USA

${ }^{115}$ National Astronomical Observatory of Japan, 2-21-1 Osawa, Mitaka, Tokyo 181-8588, Japan

${ }^{116}$ Observatori Astronòmic, Universitat de València, E-46980 Paterna, València, Spain

${ }^{117}$ School of Mathematics, University of Edinburgh, Edinburgh EH9 3FD, United Kingdom

${ }^{118}$ University and Institute of Advanced Research, Koba Institutional Area, Gandhinagar Gujarat 382007, India

${ }^{119}$ Indian Institute of Technology Bombay

${ }^{120}$ University of Szeged, Dóm tér 9, Szeged 6720, Hungary

${ }^{121}$ Tata Institute of Fundamental Research, Mumbai 400005, India

${ }^{122}$ INAF, Osservatorio Astronomico di Capodimonte, I-80131, Napoli, Italy

${ }^{123}$ University of Michigan, Ann Arbor, Michigan 48109, USA

${ }^{124}$ Abilene Christian University, Abilene, Texas 79699, USA

${ }^{125}$ American University, Washington, D.C. 20016, USA

${ }^{126}$ University of Biat ystok, 15-424 Biat ystok, Poland

${ }^{127}$ University of Southampton, Southampton SO17 1BJ, United Kingdom

${ }^{128}$ University of Washington Bothell, 18115 Campus Way NE, Bothell, Washington 98011, USA

${ }^{129}$ Institute of Applied Physics, Nizhny Novgorod, 603950, Russia

${ }^{130}$ Korea Astronomy and Space Science Institute, Daejeon 34055, Korea

${ }^{131}$ Inje University Gimhae, South Gyeongsang 50834, Korea

${ }^{132}$ National Institute for Mathematical Sciences, Daejeon 34047, Korea

${ }^{133}$ Ulsan National Institute of Science and Technology

${ }^{134}$ NCBJ, 05-400 Świerk-Otwock, Poland

${ }^{135}$ Institute of Mathematics, Polish Academy of Sciences, 00656 Warsaw, Poland

${ }^{136}$ Cornell University

${ }^{137}$ Hillsdale College, Hillsdale, Michigan 49242, USA

${ }^{138}$ Hanyang University, Seoul 04763, Korea

${ }^{139}$ NASA Marshall Space Flight Center, Huntsville, Alabama 35811, USA

${ }^{140}$ Dipartimento di Fisica, Università degli Studi Roma Tre, I-00154 Roma, Italy

${ }^{141}$ INFN, Sezione di Roma Tre, I-00154 Roma, Italy

${ }^{142}$ ESPCI, CNRS, F-75005 Paris, France

${ }^{143}$ Southern University and A\&M College, Baton Rouge, Louisiana 70813, USA

${ }^{144}$ College of William and Mary, Williamsburg, Virginia 23187, USA

${ }^{145}$ Centre Scientifique de Monaco, 8 quai Antoine Ier, MC-98000, Monaco

${ }^{146}$ Indian Institute of Technology Madras, Chennai 600036, India

${ }^{147}$ INFN Sezione di Torino, Via P. Giuria 1, I-10125 Torino, Italy

${ }^{148}$ Institut des Hautes Etudes Scientifiques, F-91440 Bures-sur-Yvette, France

${ }^{149}$ IISER-Kolkata, Mohanpur, West Bengal 741252, India 
${ }^{150}$ Whitman College, 345 Boyer Avenue, Walla Walla, Washington 99362, USA

${ }^{151}$ Scuola Normale Superiore, Piazza dei Cavalieri 7, I-56126 Pisa, Italy

${ }^{152}$ Université de Lyon, F-69361 Lyon, France

${ }^{153}$ Hobart and William Smith Colleges, Geneva, New York 14456, USA

${ }^{154}$ Università degli Studi di Firenze, I-50121 Firenze, Italy

${ }^{155} \mathrm{OzGrav}$, Swinburne University of Technology, Hawthorn VIC 3122, Australia

${ }^{156}$ Dipartimento di Fisica, Università degli Studi di Torino, I-10125 Torino, Italy

${ }^{157}$ Janusz, Gil Institute of Astronomy, University of Zielona Góra, 65-265 Zielona Góra, Poland

${ }^{158}$ University of Washington, Seattle, Washington 98195, USA

${ }^{159}$ SUPA, University of the West of Scotland, Paisley PA1 2BE, United Kingdom

${ }^{160}$ King's College London, University of London, London WC2R 2LS, United Kingdom

${ }^{161}$ Indian Institute of Technology, Gandhinagar Ahmedabad Gujarat 382424, India

${ }^{162}$ Indian Institute of Technology Hyderabad, Sangareddy, Khandi, Telangana 502285, India

${ }^{163}$ International Institute of Physics, Universidade Federal do Rio Grande do Norte, Natal RN 59078-970, Brazil

${ }^{164}$ Villanova University, 800 Lancaster Ave, Villanova, Pennsylvania 19085, USA

${ }^{165}$ Andrews University, Berrien Springs, Michigan 49104, USA

${ }^{166}$ Università di Siena, I-53100 Siena, Italy

${ }^{167}$ Trinity University, San Antonio, Texas 78212, USA

${ }^{168}$ Van Swinderen Institute for Particle Physics and Gravity, University of Groningen, Nijenborgh 4, 9747 AG Groningen, Netherlands

${ }^{169}$ Colorado State University, Fort Collins, Colorado 80523, USA

${ }^{170}$ Department of Physics, University of Texas, Austin, Texas 78712, USA

${ }^{\dagger}$ Deceased. 